\title{
The AIM2 Inflammasome: sensor of pathogens and cellular
}

\section{perturbations.}

Authors: Jérôme Lugrin ${ }^{1}$ Fabio Martinon ${ }^{2 *}$

\author{
Affiliations: \\ ${ }^{1}$ Service of Adult Intensive Care Medicine, Lausanne University Hospital, Epalinges 1066, \\ Switzerland. \\ ${ }^{2}$ Departement of Biochemistry, University of Lausanne, Epalinges 1066, Switzerland. \\ *Correspondence to: \\ Fabio Martinon, \\ Departement of Biochemistry \\ University of Lausanne, \\ Ch. Des Boveresses 155 \\ Epalinges 1066, Switzerland \\ Phone: +41-21-692.5695 \\ Fax: +41-21-692.5705 \\ Email: Fabio.Martinon@unil.ch
}

Conflicts of interest statement: The author discloses no conflicts of interest.

Running title: the AIM2 inflammasome

Keywords: Inflammasomes, DNA sensors, Nuclear envelop stress, DNA damage, Innate Immunity

Words count: 13245

Number of Figures: 4 


\section{Summary (250)}

Recognition of pathogens and altered self must be efficient and highly specific to orchestrate appropriate responses while limiting excessive inflammation and autoimmune reaction to normal self. AIM2 is a member of innate immune sensors that detects the presence of DNA, arguably the most conserved molecules in living organisms. However AIM2 achieve specificity by detecting altered or misslocalized DNA molecules. It can detect damaged DNA, and the aberrant presence of DNA within the cytosolic compartment such as genomic DNA released into the cytosol upon loss of nuclear envelope integrity. AIM2 is also a key sensor of pathogens that detects the presence of foreign DNA accumulating in the cytosol during the life cycle of intracellular pathogens including viruses, bacteria and parasites. AIM2 activation initiates the assembly of the inflammasome, an innate immune complex that leads to the activation of inflammatory caspases. This triggers the maturation and secretion of the cytokines IL-1 $\beta$ and IL-18. It can also initiate pyroptosis, a proinflammatory form of cell death.

The AIM2 inflammasome contributes to physiological responses and diseases. It is a key player in host defenses, but its deregulation can contribute immune-linked diseases, such as autoinflammatory and autoimmune pathologies. Moreover, AIM2 may play a role in cancer development.

Recent studies have shown that the detection of self-DNA species by AIM2 is an important factor that contributes to diseases associated with perturbation of cellular homeostasis. Thus, in addition of being a sensor of pathogen associated molecular patterns (PAMPs), the AIM2 inflammasome is emerging as a key guardian of cellular integrity. 


\section{INTRODUCTION}

Pathogens as well as signals triggered by cellular perturbations engage innate immune programs. These responses use a set of germline-encoded pattern recognition receptors (PRRs) to limit the spreading of pathogens and restore tissue integrity and function. Each PRR functions by recognizing specific molecular signatures. If these signals originate from the microbial world they are referred to as pathogen-associated molecular patterns (PAMPs) or microbe-associated molecular patterns (MAMPs). If the PRRs detect selfsignatures, these signals are termed danger associated molecular patterns (DAMPs) or stress signals also known as alarmins (1). These stress signals can be generated by the loss of cellular integrity. The term homeostasis-altering molecular processes (HAMPs) has been proposed to define pro-inflammatory alterations of cellular homeostasis (2). Different types of PRR have evolved to detect a limited number of signatures. The best characterized are the Toll-like receptors (TLRs), the NOD-like receptors (NLRs), the C-type lectin receptor (CLRs), the RIG-I-like receptors (RLRs) and the AIM2-like receptors (ALRs). Although these different families differ in their overall structure, they converge on similar signal transduction cascades that eventually trigger the activation of pro-inflammatory transcription factors such as type I interferon and NF-kB. A group of NLRs and ALRs can form inflammasomes. These protein complexes form signaling platforms that harbor a proteolytic activity at their core. This enzymatic activity leads to the activation of pyroptosis-mediated cell death and/or the release of active IL-1 $\beta$ and IL-18. These two cytokines engage the transmembrane receptors IL-1R and IL-18R to promote inflammation via the activation of NF- $\mathrm{KB}$ transcriptional programs through the activation of MyD88 a key signaling adaptor in innate immunity (3). 
The proinflammatory transcription factors such as type I IFN and NF- $\mathrm{kB}$ pathway regulate cytokines and chemokines production thereby promoting leukocyte recruitment. These factors also regulate feedback controls of inflammation orchestrating the magnitude and duration of immune responses. Deregulation of these signals can contribute to diseases by promoting uncontrolled inflammation. PRRs activation are often involved in the initial steps eliciting deregulated inflammation. Defining the mechanisms by which PRRs are activated and regulated is therefore a key step in understanding how deregulated inflammation is initiated in several conditions and diseases.

Here we will review the biology of AIM2, an inflammasome-forming PRR. We will discuss its ability to detect both self and non-self DNA and how this pathway contributes to diseases.

\section{AIM2 DEFINES A NEW CLASS OF INNATE IMMUNE SENSORS}

\subsection{AIM2 structural organization}

AIM2 and the ALRs are structurally defined by two main domains; the HIN domain and the pyrin domain (PYD). The HIN domain is present in one copy in AIM2, but some ALRs such as IFI16 harbors two HIN domains at the C-terminus. This domain has structural similarities to the oligonucleotide/oligosaccharide binding (OB)-folds (4). The OB fold consists of five or six-stranded $\beta$-barrel formed by 70-80 amino acid residues. The strands are connected by loops of varying length that form the ligand-binding region of the protein. OB fold can bind oligosaccharides, oligonucleotides, proteins and metal ions. Typically, OB folds are found in proteins that interact with single stranded DNA (ssDNA) such as BRCA2, a tumor suppressor involved in the repair of DNA damages, that binds ssDNA to 
initiate homologous recombination. Based on amino-acid sequence similarities, the HIN domains within ALRs have been shown to recognize DNA and have been classified in three distinct sub-classes, HIN-A, HIN-B and HIN-C (5). It has been proposed that different subclasses may differ in function or may bind DNA with different affinities (6). The PYD domain that characterizes the N-terminus of the ALRs is a member of the death fold structures formed by six coiled alpha helices arranged in a "Greek-key" fold $(7,8)$. This fold is commonly found in proteins involved in apoptosis or inflammation and participates in protein-protein interactions leading to the formation of large signaling complexes. Death folds commonly bind to other death-fold containing domains of the same type through homotypic interactions. Therefore PYD domain proteins interact with other PYD domain proteins via highly specific PYD-PYD interactions. Upon activation, AIM2 binds and recruits the adaptor protein apoptosis-associated speck-like protein containing a CARD (ASC) via PYD-PYD interactions (9-11). This interaction contributes to the initial steps of inflammasome assembly.

\subsection{The ALRs family has undergone recent and rapid evolution}

ALRs are exclusively present in placental mammals and marsupial genomes (12). Phylogenetic analyses have shown that mammalian ALRs have evolved considerably, leading to diversification of the repertoire (13). Except for AIM2 that is possibly the most conserved ALR, no evident orthology is detected between the thirteen predicted mouse and the four human ALRs. Other mammals such as cow have only one predicted ALR in the genome (12). Such a remarkable diversity among relatively close species has been observed for genes involved in innate immunity (14). This was noted for the NLRP family, 
another group of PRR forming inflammasomes (15). It is likely that such diversity may reflect selective pressure placed on these genes by pathogens (16). While AIM2 appears to be able to recognize DNA molecules independently of the sequence, it is possible that other ALRs may detect specific sequences or modifications that would identify the pathogenic DNA encountered. This hypothesis could explain the selection pressure observed for ALRs family member and the relative conservation of AIM2. Analysis of AIM2 genome suggested that AIM2 expression is deficient in the genome of some mammals such as cow, sheep, dolphin, pig, cat and dog. This may indicate that through amplification and duplication processes other ALRs may have fulfilled AIM2 function in these mammals.

Interestingly, analysis of over ten bat genomes revealed that all bats had lost the entire ALRs repertoire while other NLR innate immune sensors such as NLRP3 were conserved (17). The evolutionary events that specifically removed the ALRs genes from bats genomes are still unclear. Bats are the only mammals capable of flight. This process is very demanding on the metabolic machinery and may cause the accumulation of harmful metabolic byproducts that can affect cellular homeostasis and integrity. Inflammatory and repair pathways could be induced as a response to the release of these stress signals. Thus, it has been suggested that the loss of ALRs could be part of an adaptation during the evolution of flight (17). While this hypothesis is of interest, it also possible that the loss of ALRs in bats may reveal an evolutionary arms race that occurred between pathogens and this host. Bats are well-known reservoir of numerous pathogens including DNA viruses. It is possible that the loss of ALRs in these organisms is the result of a coevolution between bat innate immune sensors and a pathogen. While the mechanisms that triggered the loss of ALRs in bats are still unclear, it is likely that the loss of ALRs 
facilitates evasion of host immune responses by pathogens, explaining how these organisms became suitable hosts of many viruses and other disease agents.

\subsection{AIM2 is induced by type I interferon}

Using subtractive cDNA selection, AIM2 (that refers to Absent In Melanoma 2) was identified as a gene that was differentially expressed in a model of melanoma (18). While the function of this protein was unknown at the time, hints on its function emerged from studies that characterized its localization and pattern of expression. AIM2 is localized mostly within the cytoplasm of cells, suggesting that it was likely not involved in directly regulating transcriptional processes (19). Expression studies showed that, at basal, AIM2 expression is mostly detected in the spleen, in small intestine and in peripheral blood (12). However AIM2 expression can rapidly be induced. In fact, most ALRs expression is upregulated upon treatment that promote type I IFN. This observation defined early research on this family and linked ALRs proteins with inflammation and immunity (20-22). Studies in mice confirmed that most ALRs are inducible by type I IFN signaling (13).

Interferons are important mediators of host defenses against invading bacteria and viruses and can also contribute to autoinflammation. Type I IFN are produced by PRR upon detection of self-molecules or microbial-derived ligands. For example, RIG-I detects microbial RNAs and possibly endogenous RNAs (23). The cGAS-STING pathway triggers IFN- $\beta$ production in response to a variety of DNA originating form bacterial, viral, parasitic, or self-origins. In response to DNA, the cGAMP synthase (cGAS) produces Cyclic-GMP-AMP (cGAMP). This molecule, then, directly interacts with STING leading to its activation (24). In addition, interferons can cooperate with inflammasomes to 
orchestrate immune responses (25). While inflammasome engagement per se does not require transcriptional events, several inflammasomes such as NLRP3 and AIM2 require an initial pro-inflammatory signal to promote the expression of inflammasome platforms and substrates. This priming phase, also referred to as "signal 1", is mostly mediated by NF- $\mathrm{KB}$ transcriptional activation for NLRP3 and type I IFN induction for AIM2 (26). Inflammasome activation by AIM2 therefore rely or is amplified by the activation of a Type I interferons, a response that is typical of viral infections. It is worth noting that cytosolic DNA can trigger both signal 1 for AIM2 via cGAS-STING and signal 2 that leads to the post-translational assembly of the AIM2 inflammasome, further demonstrating that cytosolic DNA engages multiple parallel pathways that cooperate to maximize inflammatory responses. This cooperation is also exemplified by the role of several players involved in inflammasome activation. For example, the interferon-inducible protein, IRGB10 is required for activation of the DNA-sensing AIM2 inflammasome upon infection with Francisella novicida and contributes to the caspase-11 and NLRP3 inflammasome upon infections Gram-negative bacteria (27).

\section{AIM2 ACTIVATION LEADS TO INFLAMMASOME ASSEMLY}

\subsection{Inflammasomes are caspase-activating machines}

Inflammasomes are high-molecular weight cellular complexes that, by definition, assemble to recruit and activate inflammatory caspases (28). Inflammatory caspases namely human and mouse caspase-1, human caspase-4 and caspase-5 and mouse caspase-11, are cysteineaspartic proteases that are characterized in mammals by the presence of a caspase recruitment domain (CARD) at the $\mathrm{N}$-terminus and a C-terminal portion that is typical of 
caspases. The modular structure of inflammatory caspases resemble that of apoptotic initiator caspases. These enzymes are synthesized as inactive zymogens (pro-caspases) that can be swiftly activated following an appropriate stimulus. This activation is posttranslational and required proximity induced dimerization of the pro-caspases, leading to the stimulation of the enzymatic activity by the caspase dimer and the subsequent processing of the pro-caspase into a small subunit and large subunit (29). Apoptosome formation is among the best-characterized mechanisms mediating activation of initiator caspases. A clear delineation of the sequential events required for apoptosome formation have been described (30). At the initiation stages of apoptosis, mitochondrial cytochrome $\mathrm{c}$ is released into the cytosol. Then, cytochrome $\mathrm{c}$ binds apoptotic protease activating factor-1 (Apaf-1), triggering a conformational change that results in its oligomerization and recruitment of caspase- 9 . The resulting apoptosome facilitates the autocatalytic cleavage of the pro-caspase-9 that remains active and associated with the apoptosome (31). Studies on the apoptosome inspired the experiments that lead to the initial discovery of the inflammasome. The inflammasome was first detected in a cell-free assay that was designed to study the apoptosome complex (28). The term inflammasome was coined to acknowledge the apparent similarity of the complex with the apoptosome and to indicate that its main function is the activation of inflammatory caspases such as caspase-1. In contrast to the apoptosome that is a well-conserved and unique complex, inflammasomes can be assembled by several initiator PRRs that can detect different stimuli. However most classical inflammasomes share common mechanisms of activation that were initially described in the context of NLRP3 inflammasome activation $(32,33)$. 
The NLRP3 inflammasome is formed by the recruitment of the adaptor ASC through PYDPYD interactions and subsequent recruitment of caspase- 1 via the caspase CARD and the CARD of ASC. As a result of the initial ASC oligomerization within the inflammasome, ASC can further auto-assemble into large filament structures. This process is referred to as "prion-like" polymerization. It dramatically amplifies the inflammasome assembly by virtually engaging all ASC molecules into one active molecular structure (34). This amplification was described for several signalling pathways that use the death fold (such as PYD and CARD) as a recruitment and activation domain. Most of these pathways are involved in innate immunity and may have evolved to enable a robust and rapid response to an insult (35). Prionisation of the signal has been observed for all ASC-containing inflammasome tested so far including the AIM2 inflammasome (36).

Within the AIM2 inflammasome, the PYD of AIM2 drives both filament formation and may facilitate ligand binding (37). The AIM2 PYD oligomers orchestrate the filamentous structure of the downstream ASC filaments and subsequent AIM2 inflammasome signaling (Figure 1). These filaments form a multitude of caspase-1 activation sites, thereby amplifying inflammasome signaling (38).

\subsection{Inflammasomes mediated IL-1 $\beta$ and IL-18 production.}

In contrast to apoptotic caspases that have multiple substrates described so far, inflammatory caspases have only a few bona fide substrates that have been characterized. Inflammatory caspases are termed 'inflammatory' because the main caspase-1 substrates identified to date are proIL-1 $\beta$ and proIL-18, two structurally related cytokines that contribute to inflammation. IL-1 $\beta$ is a pleiotropic cytokine that acts on multiple cell types 
to drive inflammatory responses (39). At the systemic level, IL-1 $\beta$ elicits a fever response by acting directly on the hypothalamic temperature regulation centre (40). IL-1 $\beta$ is mostly produced by cells of the innate immune system and signals by binding to the IL1 receptor type 1 (IL1R1) complex. Once engaged, IL1R1 and its co-receptor IL1 receptor accessory protein (IL1RAP) recruit signalling adaptor and effector proteins that will trigger the production of inflammatory mediators. Similarly, IL-18 signals by engaging the IL-18R complex. Proteins such as MyD88 and IRAK4 are key components used by the proximal signalling complexes triggered by the IL-1R the IL-18R pathways as well as the TLRs (41). This similarity clearly indicates that the inflammasome-IL-1 $\beta /$ IL-18 system and the TLRs engage analogous response at the molecular level and suggest these innate pathways are variations that originate from a common ancestor pathway.

\subsection{Inflammasomes mediated Pyroptosis}

Another important target of inflammasome proteolytic activity leads to the activation of a pro-inflammatory type of cell death termed pyroptosis $(42,43)$. Pyroptosis was first observed in macrophages infected with intracellular bacteria $(44,45)$. In infected macrophages, caspase-1 deficiency impaired pyroptosis. In addition to caspase-1, other pro-inflammatory caspases such as caspase-11 have robust pyroptosis-inducing properties. Inflammatory caspases-mediated cell death, differs from caspases-mediated apoptosis in that it disrupts membranes in a way that triggers the release of the cytosolic content into the extracellular milieu. This leads to the exposure of danger signals and several proinflammatory mediators that can initiate or amplify immune responses. By disrupting membrane boundaries, pyroptosis has also been proposed to contribute to the passive release of leaderless cytokines such as mature IL-1 $\beta$ (46). 
AIM2 mediated pyroptosis has been shown to be caspase-1-dependent. However, in caspase-1 deficient mice, AIM2 activation promotes the activation of the proapoptotic caspase-8, leading to a caspase-1 independent cell death, by a process that resemble apoptosis (47). It is therefore likely that in the context of caspase-1 inhibition; inflammasome platform can engage alternative routes to trigger cell death, similarly to apoptosis processes that can elicit necroptosis in absence of caspases $(48,49)$.

Recently, a family of proteins named Gasdermins have emerged as key mediators of inflammatory caspases-mediated cells death (50-52). Caspase-1 or caspase-11 have been shown to cleave Gasdermin D (GSDMD). This releases the N-terminal portion of the Gasdermin that then polymerizes at the plasma membrane forming cytotoxic pores. These pores disrupt cellular integrity and result in cell death with typical feature of pyroptosis (Figure 2).

\section{AIM2 DETECTION OF DNA}

Transfection of host, bacterial and viral DNA, beside its effects on the transcriptional activation type-I IFN, has been shown to initiate a cascade leading to the proteolytic maturation of IL-1 $\beta$. These DNA molecules relied on an ASC-dependent but NLRP3independent inflammasome (53). The use of an orthogonal proteomic-genomic screen in mouse cell lines, identified AIM2 as the main cytoplasmic DNA sensor promoting inflammasome assembly. A proteomic screen for DNA-binding proteins that interacted with IFN- $\beta$-stimulating dsDNA was coupled to a genomic screen for IFN- $\beta$ regulated genes. The results of these screens were crossed and several ALR proteins were identified as candidates of interest. Among them, AIM2 was found to trigger an inflammasome while 
co-expressed with ASC, caspase-1 and IL-1 $\beta$ (9). Other studies showed that the AIM2 PYD domain was necessary to interact with ASC in order to nucleate an inflammasome $(10,11)$. These studies demonstrated that AIM2 is the cytosolic sensor for dsDNA that, upon engagement, promotes the activation of caspase-1 in mouse BMDMs. In contrast, the decoy ALR protein p202 was found to repress dsDNA activation of caspase-1 (54). AIM2 cytosolic subcellular localization and its ability to detect cytosolic DNA make of this protein a bona fide cytosolic innate immune sensor.

\subsection{DNA sensors}

The ability of the immune system to respond to DNA was first discovered with the study of the endosomal toll-like receptor 9 (TLR9). TLR9 binds CpG DNA from bacteria and triggers innate immune responses via the MyD88 adaptor (55). The DEAD/H-box helicases DHX9 and DHX36 were also found to be MyD88-dependent DNA sensors that activate NF- $\mathrm{BB}$ and IRF7 in plasmacytoid dendritic cells (56). DNA-dependent protein kinase (DNA-PK), a protein complex was found to detect cytosolic DNA via the Ku70 subunit leading to the activation of type III IFNs (57). Several DNA sensors have been described to elicit a strong type I IFN response signal through the activation of the adaptor protein STING (58). STING is a master regulator of type I IFN innate immunity triggered by cytosolic DNA(59). It centralizes the signal from the DNA sensors cGAS(60, 61), DDX41(62), DNA-PK(63), MRE11(64), DAI/ZBP1 $(65,66)$ and possibly the ALR protein IFI16 and its mouse ortholog p204(67). These pathways trigger the STING-TBK1-IRF3/7 axis and subsequent transcription of type I IFNs (58). 
Compared to other DNA sensors AIM2 is specialized in inflammasome assembly rather than promoting type I IFN responses.

\subsection{AIM2 directly binds DNA molecules}

Biochemical studies indicated that AIM2 binds DNA directly and that the HIN domain was sufficient for this interaction (11). Consitently, mutations within the HIN domain impaired AIM2 ability to bind immunostimulatory dsDNA immobilized on resin (9). ALRs were described to bind dsDNA of virtually any origin including microbial, host or synthetic molecules that vary in sequence and GC content $(9-11,54,67)$. The lack of apparent specificity of the HIN-DNA interactions is supported by the nature of the HIN-DNA interactions $(68,69)$. Sensitivity of HIN-DNA interaction to ionic strength suggested the involvemnt of electrostatic attractions (68). Moreover, crystallographic analyses of DNA interaction with AIM2 and IFI16 revealed that the hydrophobic pocket of the HIN binds both strands of DNA on the sugar-phosphate backbone across major and minor grooves $(68,69)$. In absence of DNA ALRs are in an autoinhibited state with PYD and HIN domains. This suggests a machanisms of AIM2 activation in which binding of DNA to the HIN domain releases the PYD from its interaction with the HIN domain, thereby promoting PYD-PYD interaction with ASC and inflammasome assembly (70).

\subsection{AIM2 detects DNA from pathogens}

During infections with intracellular pathogens, microbial DNA can be released in the cytosol. AIM2 function as a DNA sensor has been extensively described in the context of several microbes and pathogens (71), further highlighting the role of this protein in innate immunity.

\section{AIM2 detects bacterial DNA}


Francisella tularensis $(72-74)$ and Lysteria monocytogenes $(73,75)$ were the first live bacteria identified to engage the AIM2 inflammasome. In particular F. tularensis is a poor activator of innate immune pathways and function mostly by engaging TLR2 and the AIM2 inflammasome. In contrast, Lysteria monocytogenes activates several inflammasomes and AIM2 contribution is best detected in macrophages deficient for NLRC4 and NLRP3 (75). So far, many bacterial species have been found to activate AIM2 including F. tularensis, L. monocytogenes, Streptococcus pneumoniae, species of Mycobaterium as well as Legionella pneumophila and Staphylococcus aureus. These observations have been extensively reviewed recently (71).

In order to be detected by AIM2 bacteria have the necessity to escape the vacuole and undergo bacteriolysis, leading to the exposure of bacterial DNA in the cytosol. Via activation of autocrine type I IFN signaling, Francisella subspecies novicida (F. novicida) induces the expression the transcription factor IRF1. IRF1 in turn controls the expression of IFN-inducible GTPases tremed guanylate-binding proteins (GBPs) in an autocrine manner. GBP2 and GBP5 associate with $F$. novicida present in the cytoplasm and trigger bacteriolysis $(76,77)$. GBP2 and 5 cannot rescue inflamamsome activation in type I IFN receptor-deficient macrophages $(76,77)$ indicating that additional IFN-induced proteins act in collaboration with GBP2 and GBP5 to drive bacterial lysis. Indeed, the IFN-inducible protein IRGB10 has recently been described as a crucial partner for GBPs for killing of intracellular bacteria (27). IRGB10 colocalizes with GBPs at the membrane of intracellular bacteria that escaped in the cytosol. It is present to bacterial membrane sites where membrane integrity is lost. Moreover, sequence analysis of IRGB10 indicates the presence of amphipathic helices and myristoylation motifs that may account for its membrane 
disrupting properties. This however needs to be experimentally characterized. $\operatorname{Irg} b 10^{-/-}$ mice, similar GBPs-, AIM2- and Caspase-1-deficent mice, are less resistant to F. novicida infection, exhibit higher bacterial loads and less IL-18 production than control mice. Moreover, IRGB10 was not only shown to favor the release of bacterial DNA in the cytosol to fully activate AIM2 inflammasome, it also favors the release of LPS from bacterial membrane to activate Caspase-11 and possibly other inflammasomes (27).

\section{AIM2 detection of Viruses}

Given its abilities to recognize non-specifically dsDNA structures, one may picture AIM2 to be a broad sensor of DNA viruses. However, despite the fact that AIM2 can detect viral DNA its contribution to viral immunity is still unclear. Mouse cytomegalovirus (MCVM), vaccinia virus and human papilliomavirus have been shown to induce AIM2 inflammasome $(11,73,77,78)$. Mechanism by which viral DNA is liberated and exposed to AIM2 in the cytosol is not clearly defined. Viruses have been shown to evade AIM2 sensing possibly by inhibiting the ability of AIM2 to interact with released DNA. A recent study reported that Epstein-Barr virus (EBV) may activate AIM2 in THP1 cells (79). Two studies recently showed that influenza virus (IAV), a single stranded RNA virus, could trigger the release of endogenous DNA from damaged lung tissue possibly leading to AIM2 activation $(80,81)$. One study stated that AIM2 dampened detrimental inflammation in the lung microenvironment of mouse infected with IAV and that AIM2-deficient mice had exacerbated immune responses to IAV (80). The other study reported that AIM2-deficient mice infected with IAV infection had less lung tissue damage and improved survival ( 80 , 81). While these findings may seem contradictory, they both suggest that AIM2 can detect 
host-DNA released in the context of viral infections. The physiological role of this detection and the discrepancies observed in the model tested await further studies.

\subsection{AIM2 detects endogenous self-DNA upon disruption of nuclear envelope integrity}

The nuclear envelope (NE) defines a cellular compartment that isolates the nucleus and its content from the cytoplasm. The NE inner and outer membranes are highly dynamic structures riddled with proteins that regulate their functions (82). The nuclear lamina is a meshwork of proteins lining the inner nuclear membrane, crucial to NE integrity, transmission of mechanical forces, chromatin organization and gene transcription (83). Hereditary mutations promoting an impairment of lamin A maturation, an essential component of the nuclear lamina, cause a family of diseases called laminopathies. These diseases have a large variety of clinical symptoms including cardiac muscular dystrophy, lipodystrophy, diabetes, and premature aging.

Pathologies such as laminopathies, viral infections and cancer are characterized by transient alterations of NE homeostasis $(82,84)$. Defects in NE homeostasis may materialize as large blebs of herniating DNA and lamina gaps as observed upon infection with HIV (85) or as transient nuclear rupture as observed in cultured fibroblasts from laminopathy patients (86). Phenotypically resembling laminopathic defects, cancer cells also exhibit defaulted lamin A expression with a nuclear morphology exhibiting transient nuclear envelope ruptures $(87,88)$.

A consequence of transitory loss of nuclear compartmentalization is an exchange of materials between nucleus and cytoplasm. This phenomenon was observed during spontaneous NE ruptures occurring in laminopathic fibroblasts or in migrating cells forced 
through microscopic pores or glass capillaries $(86,89)$. Transient nature of NE ruptures without subsequent elimination of damaged cells indicate the presence of NE repair system that remained for long not understood (90). However quality control and repair mechanisms of ruptured NE have been recently described (91). Notably, innate immune and cancer cells were recently shown to involve the ESCRT (endosomal sorting complexes required for transport) III system to repair NE after NE ruptures occurring when cells migrates through small pores $(89,92)$. This suggests a physiological role of these processes, for example in the context of cellular migration.

During NE rupture, transient exposure of nuclear components within the cytosolic compartment raises the question of the possible activation of cytosolic DNA sensors. Cells forced through tiny pores exhibited cGAS localization at sites of NE ruptures indicating that cGAS is binding DNA at these sites (89). Participation of cytosolic innate immune receptors in NE-stress and transient membrane rupture was recently confirmed in a model of pharmacological perturbation of the nuclear envelope structure (93). Lamin A maturation was found to be inhibited by the anti-viral drug nelfinavir, causing pertubations in nuclear architecture and transient exchanges of nuclear and cytoplasmic contents. As consequence of exposure of nuclear DNA in the cytoplasm, AIM2 and cGAS cytosolic DNA sensors were activated (93) (Figure 3). These results further emphasized on a possible contribution of stress of the NE and its associated inflammatory pathways to diseases with transient NE ruptures, including laminopathies and cancers. Yet the physiological relevance of this contribution remains to be investigated.

\subsection{AIM2 detects DNA damage within the nucleus}


Besides its well-described ability to detect dsDNA within the cytosol, AIM2 has been recently proposed to detect DNA damage directly within the nucleus (94). In a mouse model of subtotal body exposure to ionizing radiation with gastrointestinal toxicity, inflammasome-deficient mice were found to be more resistant to irradiation. AIM2 was identified as the only inflammasome sensor responsible for resistance to irradiationinduced intestinal toxicity in this model. Irradiation toxicity was mediated by pyroptosis of epithelial intestinal cells. Notably, drugs such as doxorubicin and etoposide that trigger DNA double-strand breaks (DSB) also promoted AIM2-mediated cell death. In contrast UV irradiation, that mediates single-strand breaks, did not rely on AIM2 to promote cytotoxicity. Interestingly AIM2 colocalized with $\gamma$-H2Ax at sites of DSB in irradiated BMDM. Moreover, ASC specks were also identified within the nucleus upon DNA damage, indicating that the AIM2 inflammasome can assemble and function within nuclei. Whether nuclear inflammasomes have different proteolytic substrates than cytosolic inflammasome is a possibility that needs to be investigated. While IL-1 $\beta$ localization within the nucleus was detected before (95), the pyroptosis-mediating factor Gasdermin D was reported to be exclusively cytosolic (96). It is possible that Gasdermin D localizes in the nucleus of epithelial cells undergoing DNA damage, alternatively AIM2 could uses a different pathway to promote pyroptotic cell death upon DNA damage.

\subsection{AIM2 detects released self-DNA secreted by exosomes}

Besides sensing cell intrinsic dsDNA, AIM2 in macrophages and dendritic cells has recently been proposed to sense self-DNA delivered by exosomes produced by intestinal epithelial cells exposed to chemotherapeutic agents (97). In colon cancer patients treated with the cytotoxic chemotherapeutic agent CPT-11 (Irinotecan), the severity of side-effects 
such as diarrhea correlated with serum levels of dsDNA (97). Moreover in a mouse xenograft model in which MC-38 murine colon carcinoma cells were injected prior to Irinotecan administration to mice, small intestine shortening and dsDNA increase in peritoneal lavage was observed. Released dsDNA could induce IL-1 $\beta$ maturation and inflammasome assembly when transfected in BMDM and BMDCs. This activation was abrogated in AIM2-deficient BMDM and BMDC (97). Further studies showed that the main source of dsDNA released by cells treated with an active form of CPT-11 was present within exosomes. These DNA-loaded exosomes were taken up by BMDM. In mice, chemical inhibition of exosomes generation as well as AIM2-deficiency reduced CPT-11 mediated intestinal damages caused by inflammasome activation. Exosomes may therefore act as vehicle for the delivery of inflammatory DNA, possibly protecting such DNA from degradation by extracellular nucleases.

\subsection{AIM2 detects DNA vaccines}

The ability of AIM2 to sense dsDNA from extrinsic origin makes it a good candidate as DNA vaccines sensor. In vitro, IL-1 $\beta$ production as well as pyroptosis were found to be mediated by AIM2 in BMDM transfected with a DNA vaccine. Moreover, vaccine immunogenicity was greatly impaired in AIM2 deficient mice as reflected by decreased antigen-specific IgG, antigen-specific splenic B cells and IFN- $\gamma$-secreting $\mathrm{T}$ cells. One

notable point is that in this model, immunogenicity did not depend on IL-1 and IL-18 signaling since IL-1R- and IL-18R-deficient mice mounted normal responses. Interestingly, AIM2 expression was reported to be required in both hematopoietic and nonhematopoeitic lineages in order to mount a full response to DNA vaccine (98). The 
mechanisms and cell types involved remain to be determined. AIM2 deficiency decreased type I IFN production in AIM2-deficient mice treated with DNA vaccine. This observation is puzzling and needs to be further investigated. The cGAS/STING pathway has been described to significantly contribute to DNA vaccine-mediated immunogenicity $(59,99)$. However, AIM2 inflammasome activation has been reported to antagonize the STING pathway (100). This antagonism relies on cleavage of cGAS by caspase-1(101). It is therefore possible that the regulation of type I interferon and the activation of the AIM2 inflammasome occurs at a different time or in a different cell type in the course of DNA vaccination.

Because of its ability to detect DNA vaccines, increased AIM2 expression was considered as an attractive strategy to boost DNA vaccination protocols. Three recent studies showed that immunization of mice against coxsackie virus B3 (CVB3) using AIM2 as a mucosal adjuvant had beneficial effects. Indeed compared to mice immunized with CVB3-specific chitosan-pVP1, co-immunization of mice with CVB3-chitosan-pVP1 and chitosan-pAIM2 conferred better resistance to CVB3-induced myocarditits, increased secretory IgA (102), increased CVB3-specific $\mathrm{T}$ cells responses (103) and increased counts of CD8 ${ }^{+}$memory $\mathrm{T}$ cells (104).

\section{REGULATION OF AIM2}

Inappropriate or chronic activation of inflammasomes can lead to diseases displaying features of autoinflammation (105). Regulation of the engagement, the intensity and the duration of the response is therefore critical to optimize the reaction while minimizing possible side effects of inflammation. While complete understanding of the overall 
circuitry of inflammasomes regulation is still missing, a few mechanisms impacting AIM2 activity have been described.

\subsection{Regulation of ligand availability}

Among the strategies that regulate aberrant engagement of DNA sensors such as AIM2, organisms have developed mechanisms to compartmentalize DNA and to degrade it when mislocalized. This process limits inflammation triggered by apoptotic cells, and reversetranscribed DNA from endogenous retro-elements $(106,107)$. The physiological relevance of these mechanisms is exemplified by the fact that deficiencies in nucleases, such as DNase II or Trex1, leads to an accumulation of self-DNA that ultimately reaches the cytosol where it drives autoinflammatory diseases.

Certain DNA sequences such as the TTAGGG repeat, regularly found in mammalian telomeric DNA, have been shown to display anti-inflammatory activities. Synthetic oligodeoxynucleotides with this sequence have been shown to inhibit AIM2 inflammasome activation (108), indicating that specific sequence may interfere with ALRs activations. It is possible that these sequences compete for AIM2 binding thereby blocking the recognition of pro-inflammatory DNA sequences. Whether similar sequences from endogenous sources or from pathogens can efficiently regulate AIM2, is an interesting hypothesis that has not been demonstrated yet.

\subsection{Regulation AIM2 by encoded decoy proteins}

Several proteins have been proposed to interfere with the assembly of inflammasomes. Decoy proteins encoding PYD or CARD only domains are believed to inhibit inflammasome assembly by interfering with PYD-PYD or CARD-CARD homotypic 
interactions and thereby blocking the recruitment of adaptor and effector proteins to the complex (109) (Figure 4). Similar to the repertoire of ALRs and NLRPs, PYD-only regulators (POPs) and CARD-only regulators (COPs) are poorly conserved in mammals. In humans, three POPs have been identified whiles two predicted POPs sequences are present in the mouse genome. Interestingly these human and mouse POPs share weak homology and are not orthologs making their studies in animal models challenging. Initial evidences indicating that POPs negatively regulate inflammasomes came from the identification of viral POPs. M13L a poxvirus-encoded POP was shown to interact with ASC to inhibit inflammasome engagement (110). Human POP3 was identified to bind AIM2. ASC and POP3 therefore compete for interaction with AIM2 thereby dampening inflammasome activation (111). Mice engineered to constitutively express human POP3 within macrophages have impaired AIM2-activation and defective ability to handle viral DNA-induced host defense in vivo (111). This phenotype resembles the phenotype observed in AIM2 deficient animals (73), and clearly indicates that these proteins can impair AIM2 activation. However, in this model, POP3 expression was artificially enforced with a strong promoter. Whether POP3 is induced in physiological conditions, and whether it regulates inflammasomes in human, remains to be demonstrated.

Three COPs have been identified; these proteins are all encoded within the caspase-1 locus in human and are absent from the mouse genome. They are all very homologous to the caspase-1 CARD and are believed to be the result of recent duplication of the caspase-1 gene $(109,112)$. COPs all are predicted to function as caspase-1 decoy proteins that that blocks the recruitment of caspase-1 to inflammasomes. However the physiological relevance of these putative regulators is still uncharacterized. 
Other decoy proteins in the pathway have been described within the ALR family. In mice, the ALR p202 lacks the PYD domain and has been shown to inhibit AIM2 activation (54). Mouse p202 harbors two HIN domains, one has been shown to interact with dsDNA while the other one has affinity for AIM2 HIN domain (113). It is possible that p202 functions by competing for DNA and therefore may block several innate immune sensors of cytosolic DNA. However, p202 has little effect on the activation of type I IFN by the cGAS-STING pathway suggesting that it may directly impact AIM2. This was confirmed by structural studies that demonstrated the p202 through its second HIN domain interact with AIM2 to prevent its oligomerization and subsequent recruitment of caspase-1 $(113,114)$.

\subsection{Other regulators of AIM2}

Beyond regulators that impact ligand accessibility and impair the assembly of inflammasomes, AIM2 protein levels can be directly regulated. The tripartite motif 11 (TRIM11) was reported to be a key negative regulator of the AIM2 inflammasome. Upon activation, TRIM11 interacts with AIM2 leading to the recruitment of the autophagic cargo receptor $\mathrm{p} 62$. The recruitment of this machinery to AIM2 leads to AIM2 degradation via selective autophagy (115). These findings demonstrate that post-translational modifications contribute to inflammasomes regulation and may be key at regulating the intensity and duration of the inflammatory responses (116).

AIM2 signaling is regulated by pathogens. For example, Mycobacterium tuberculosis dampens proinflammatory signals triggered by microbial DNA within infected macrophages. Mechanistically the exact nature of this inhibition is still unknown however it was proposed to function by affecting Type I IFN (signal 1) as well as AIM2 assembly 
(signal 2) $(117,118)$. This regulatory loop was found to be dependent on the type VII secretion system (ESX-1), a system required for the translocation of $M$. tuberculosis DNA into the host cell cytosol. The fact that the same system can also trigger the release of an inhibitor of DNA signaling, may indicate that this mechanism has evolve to counteract immune response triggered by the exposure of microbial DNA within host cells.

\section{AIM2 IN HEALTH AND DISEASE}

\subsection{Role in Infectious diseases}

AIM2 role in sensing pathogens has been shown to contribute to infection in several models. Francisella tularensis live vaccine strain (LVS) and subspecies novicida, Lysteria monocytogenes, Streptococcus pneumoniae, Mycobacterium tuberculosis and bovis, Porphyromonas gingivalis, Legionella pneumophila, Staphylococcus aureus, Brucella abortus and Chlamydia muridarum have been shown to rely on this pathway (71). Several pathogens developed mechanisms to limit detection by AIM2 further highlighting the role of AIM2 in regulating immune responses. F. tularensis subspecies developed strategies to resist bacteriolysis through evolution of virulence factors preventing release of microbial products such as bacterial wall components or DNA. F. tularensis subspecies ShuS4 is more resistant to bacteriolysis induced by mitochondrial ROS (119). Deletion of RipA protein (120) or mutation of the lipid flippase MviN (121) in F. tularensis as well as absence of Sdha gene in L. pneumophiliae (122) all increased AIM2 inflammasome activation. Remarkably, F. novicida developed a clustered, regularly interspaced, short palindromic repeats-CRISPR associated-Cas (CRISPR-Cas) system strengthening bacterial envelope and conferring resistance to host sensors AIM2 and TLR2 (123). 
Recently, components of the type VI secretion system (T6SS) PdpC and PdpD encoded on the Francisella pathogenicity island and the chaperone $\mathrm{ClpB}$ were shown to be essential for phagosome escape of $F$. tularensis (124). Phagosomal rupture and AIM2 inflammasome activation depended on T6SS disassembly, which is regulated by ClpB. Strains lacking $p d p C$ and $p d p D$, normally released by T6SS, were also unable to escape phagosome and activate AIM2 but their exact function is still not characterized (124).

Surprisingly, only few DNA viruses have been reported to activate AIM2. Among them mouse cytomegalovirus (mCMV) and Vaccinia virus were the first described (73). AIM2, cleaved caspase- 1 and cleaved IL-1 $\beta$ were detected in the skin lesion of patients infected with Human papillomavirus 16 (HPV16) in region where cytosolic DNA of HPV16 could also be detected (78). Human primary keratinocytes released IL-1 $\beta$ and IL-18 when transfected with HPV16 DNA and silencing of AIM2 greatly reduced this release (78). In an ex vivo human APC model, immune-complexed adenovirus was reported to destabilize endosomal membrane via the release of protein VI during capsid disassembly promoting the release of viral DNA in the cytoplasm of the APC. Viral DNA then activated AIM2 inflammasome triggering caspase-1 cleavage, IL-1 $\beta$ maturation and GSDMD processing (125). This process has not been studied in vivo so far and whether it benefits to host or pathogen needs to be investigated. AIM2 was also reported to promote inflammationmediated renal damage in patients with Hepatitis B virus-associated glomerulonephritis (HBV-GN) (126). AIM2 was detected in glomerular endothelial and mesangial cells of patients with HBV-GN and AIM2 silencing in human mesangial cells infected with HBV led to decreased expression of IL-1 $\beta$ and IL-18 (126). The direct participation of AIM2 in HBV DNA recognition has not been investigated. Despite the well-established 
participation of AIM2 in the control of mouse CMV, the role of AIM2 in human CMV (hCMV) infection is still poorly defined. Yet, recently the hCMV protein pUL83 was discovered to interact with human AIM2 and disrupt AIM2 inflammasome activation (127) indicating that AIM2 may be regulated by the viral infection.

AIM2 has also been described to control RNA viruses including Chikungunya virus (CHINKV), West Nile virus (WNV) and Enterovirus A71 (EV-A71) $(128,129)$. AIM2 and caspase-1 genes were highly up-regulated in primary human dermal fibroblasts upon infection with CHINKV and WNV. Inflammasome inhibition reduced release of IL-1 $\beta$ and increased viral titers in CHINKV and WNV infected dermal fibroblasts (128). Likewise, EV-A71 promoted up regulation of genes encoding for AIM2 inflammasome components in SK-N-SH human neural cell line and AIM2 was found highly expressed in the central nervous system of patients infected with EV-A71. Down-regulation of AIM2 in SK-N-SH cells decreased pyroptosis upon infection with EV-A71 and augmented viral replication (129). In contrast AIM2 did not control the replication of Influenza A virus (IAV) in murine models (81). However mice lacking AIM2 had reduced lung inflammation and injury after (IAV) infection as illustrated by decreased inflammatory cells infiltrate and decreases lung tissue damage (81). The mechanisms of sensing of these RNA viruses by AIM2 remains obscure and needs to be further characterized.

A role for AIM2 in fungal infections was also proposed. NLRP3 and AIM2 are both necessary and act in concert to confer a proper immune surveillance against Aspergillus fumigatus. Indeed, intranasal infection of mice with A. fumigatus led to aggravated lung infections in NLRP3 and AIM2 double knockout mice as compared to WT or single knockout controls (130). 
Plasmodium berghei-derived hemozoin and DNA also dually engage the NLRP3 and AIM2 inflammasomes during malaria. Hemozoin crystals complex with plasmodial DNA in infected red blood cells. After phagocytosis of infected red blood cells or CpG DNA-coated synthetic hemozoin, DNA is recognized by TLR9 in the endosomal compartment of murine macrophages offering a priming and activation signal for inflammasomes (131). Hemozoin activates NLRP3 possibly by promoting lysosomal rupture involving cathepsins while plasmodial DNA contained in the endosomal compartment is released in the cytosol to activate AIM2 (131). Based on these results obtained in vitro, AIM2 should play a role in systemic inflammation during malaria infection in humans. However, its exact role on the pathophysiology of malaria is still to be characterized in vivo.

The AIM2 inflammasome is also involved in the pathogenesis of leishmaniasis in patients infected with Leishmania (V.) braziliensis (132). AIM2 and caspase-1 mRNA have been found more expressed in tissue from lesions of mucosal leishamaniasis compared to lesions from the less severe cutaneous leishamaniasis. AIM2 expression and number of IL-1 $\beta$ positive cells were also higher in lesions of patients poorly responding to therapy (132). How host- or protozoan-derived DNA activates AIM2 inflammasome in tegumentary leishmania lesions is still unclear.

Perturbation in the AIM2 pathway has been reported to deregulate the commensal microbiota, indicating that he AIM2 inflammasome is a central regulator of intestinal homeostasis (133-135). It may contribute to maintain the production of epithelial antimicrobial host defense (136), and its deregulation may contribute to disease progression in the mucosa of patients with active inflammatory bowel disease (137). 


\subsection{Autoinflammation}

The concept of autoinflammatory diseases was coined to distinguish a set of inflammatory autosomal-dominant diseases from self-directed autoimmune diseases (138). Autoinflammatory diseases do not have adaptive immune cell involvement, and are believe to be caused by hyper-activation of innate immune pathways leading to aberrant inflammation (139).

Deregulation of inflammasome pathways is a common feature in several genetically inherited autoinflammatory diseases. Patients with gain of function mutation in NLRP3 have constitutively active inflammasome. This lead to persistent IL-1 $\beta$ and IL-18 secretion by myeloid cells and subsequent episodes of systemic inflammation.

Immunotherapies aimed at reducing the amount of circulating IL-1 $\beta$ or at decreasing its ability to bind to the IL-1R complex have been proven to be very efficient in these patients at decreasing systemic inflammatory symptoms such as fever and skin rashes (140).

Autoinflammatory diseases can be triggered by the increased presence of inflammasome activating signals. Gout is characterized by a powerful inflammatory reaction stimulated by the deposition of monosodium urate crystals within joints. These crystals trigger NLRP3 inflammasome activation in macrophages thereby initiating an IL-1 $\beta$-dependent autoinflammatory reaction (141).

Similarly, increased amount of self-DNA can initiate autoinflammation by exacerbated activation of the DNA sensors pathways including the cGAS-STING pathway and the AIM2 inflammasome. This was reported for example in a mouse model of polyarthritislike disease caused by DNase II deficiency. In these mice, that show increased release of 
self-DNA within the cytosol, both the cGAS-STING pathway and the AIM2 inflammasome pathway have contributed to autoinflammation $(142,143)$.

Abundant cytosolic DNA was also observed in keratinocytes in psoriatic lesions. In this disease, cytosolic DNA, behave like a "disease-associated molecular pattern" that can elicit AIM2 inflammasome leading to IL-1 $\beta$ maturation and the autoinflammation signs observed in psoriasis patients (144)

To date, AIM2 had not been linked to inherited autoinflammatory diseases and no gain-of function mutations have been identified within AIM2 gene. This is possibly due to the fact that AIM2 oligomerization may rely on DNA binding, which may promote the assembly of inflammasomes along the DNA structure (68). This is in contrast to NLR-based inflammasome that can oligomerize independently of their ligand.

\subsection{Autoimmunity}

In addition to its role in triggering inflammation, self-DNA is also associated with typical autoimmune diseases. ALRs and AIM2 have been implicated in systemic lupus erythematous (SLE) $(145,146)$. Systemic lupus erythematosus (SLE) is a chronic autoimmune disease that can affect most organs. While this disease is characterized by the presence of autoantibodies and therefore classify as an autoimmune disease, it presents also autoinflammatory features involving innate immune pathways. Monogenic mutations in the DNA nucleases (DNASE1 and DNASE1L3), which function in the rapid clearance of DNA from apoptotic cells, drive a monogenic SLE (monoSLE) that is characterized by autoinflammation and autoimmunity including positive anti-dsDNA antibodies (147). This 
indicates that over-activation of innate immune sensors of DNA may trigger an autoinflammation that could also contribute to autoimmunity in this disease.

Deregulation of type I IFN pathways is a hallmark of SLE. In addition, genetic studies in mice suggested a role for ALRs in SLE. A polymorphism increasing the expression of ALR p202 has been associated with a major lupus-predisposing locus of NZB mice (148). While it is possible that ALRs may regulate type I IFN responses and thereby modulate disease in SLE, ALRs have been found to have a minor function at directly regulating type I IFN responses (149). Because p202 is an inhibitor of AIM2 inflammasome, it is likely that the inflammasome system is altered in this model to SLE. In line with this hypothesis, macrophages from NZB mice display altered inflammasome activation upon treatment with AIM2 agonists. In addition, these mice have an additional polymorphism within the NLRP3 gene that alters its expression and blunt the ability of macrophages to mount an NLRP3 inflammasome. While the contribution of p202 and AIM2 in SLE remains debated (150-152), it is possible that the imbalance between type I IFN responses and AIM2 activation in this model contributes to autoimmunity.

\subsection{Cancer}

Tumorigenesis and inflammation are known to be tightly intertwined. Chronic inflammation promoted by environment, microbial organisms or microbiota is a major driver of cancer (153). NLRs and ALRs have been shown to play both positive and negative roles in cancer pathogenesis by regulating immune responses, proliferation, cell death and intestine dysbiosis (154). AIM2 was initially discovered as a gene absent in human

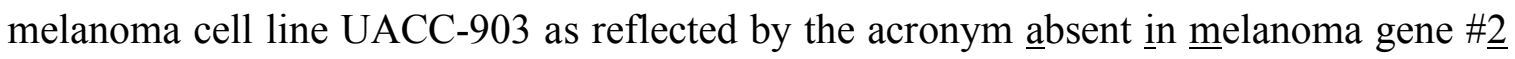
(AIM2). AIM2 was found overexpressed following reversion of the tumorigenic 
phenotype by introduction of human chromosome 6 in UACC-903. Downregulation of AIM2 protein expression was shown to play an important role in colorectal cancers (155) that display mutations in AIM2 gene (156), silencing by DNA hypermethylation (157) and specific decreased tumor tissue expression (158). Similarly, prostate cancer cells also show decreased AIM2 expression compared to cells from healthy prostate and begnin prostate hyperplasia samples (159). Two studies reported recently the loss of AIM2 expression in hepatocellular carcinoma (HCC). In liver samples from patients with HCC, AIM2 expression was decreased in cancer tissue compared to corresponding non-cancerous samples. Moreover, AIM2 expression was negatively correlated with tumor volume, grade and metastatic progression $(160,161)$. In liver, hepatitis B virus X protein (HBx) has been proposed to contribute to the downregulation of AIM2 expression both at the transcription and protein levels. Indeed, HCC patients that are HBV positive show lower AIM2 expression levels compared to HCC HBV-negative patients (161). Reconstitution of HCC cell lines with AIM2 diminished their proliferation and invasiveness while further decreasing the expression of AIM2 augmented signs of disease $(160,161)$. In this cancer type the AIM2 inflammasome was proposed to negatively impact the mTOR-S6K1 pathway. Reconstitution of AIM2 expression in HCC cell lines had an inhibitory effect on mTOR and S6K1 phosphorylation that was reversed by caspase-1 inhibition. Reconstitution of AIM2 expression also impaired tumor growth in a HCC xenograft model (160). In a different mouse model of HCC, decreased AIM2 expression led to increased number of liver tumors and lung nodules (161). AIM2 silencing increased Fibronectin 1 (FN1), a factor that could be responsible for the epithelial to mesenchymal transition 
enabling HCC metastasis (161). Whether AIM2 ability to repress FN1 depends on inflammasome assembly has not been investigated so far.

Recently, two studies characterized the role of AIM2 in a murine model of colitisassociated cancer chemically induced by azoxymethane (AOM) injection followed by dextran sodium sulfate (DSS) treatment $(162,163)$. One study used the AOM/DSS model to demonstrate that AIM2 suppression of colon tumorigenesis was independent of inflammasome formation and instead relied on the suppression of the DNA-PK-Akt axis (163). Additionally, the sporadic development of colon cancer in $A p c^{\mathrm{Min} /-}$ model, was aggravated by AIM2 deficiency. Increased Akt phosphorylation in AIM2 deficient tumors correlated with bigger tumor load. It was proposed that AIM2 could directly interact with DNA-PK, an Akt kinase activated by DNA damage, possibly limiting DNA-PK activation and subsequent Akt phosphorylation and cell proliferation (163). The second study showed that the inflammasome-independent role for AIM2 in the protection against colon cancer was mainly driven by stromal cell expression of AIM2 and not by myeloid cells $(162,163)$. Consistently they observed that AIM2-deficiency increased proliferation in vivo and Akt phosphorylation. Colonic epithelial progenitor stem cells purified from AIM2 deficient mice were more prone to form larger organoids than control mice. Moreover, aberrant activation of the WNT/ $\beta$-catenin pathway increased proliferation of epithelial stem cells in the colonic crypts of AIM2-deficient mice (162).

Conversely, AIM2 expression was increased in human cutaneous squamous cell carcinoma (cSCC) (164). Comparison of AIM2 expression levels between normal human epithelial keratinocytes and cSCC cell lines showed higher expression of AIM2 in cSCC cells. AIM2 expression correlated to the grade of the tumors, being low in normal skin, low to moderate 
in actinic keratosis (pre-cancer) lesions, moderate to high in primary cSCC tumors and high in invasive cSCC tumors. This occurred both in sporadic $\mathrm{cSCC}$ and $\mathrm{cSCC}$ associated with organ transplant (164). Pathway analysis of gene expression profiles after decreasing AIM2 in cSCC cell lines revealed alterations in pathways controlling cell cycle, proliferation and invasiveness. In vivo, decreasing AIM2 expression in human $\mathrm{CSCC}$ cells prior to xenograft transplantation in SCID mice promoted a reduction of tumor volume, cell proliferation and tumor vascularization (164). Of note, the implication of inflammasome assembly in this tumor type has not been investigated yet. Other cancers, associated with viral infections, such as Epstein-Barr virus (165) or human papillomavirus (166) also exhibited increased AIM2 expression. Altogether these studies demonstrate that AIM2 has a key role in regulating tumorigenesis, however the mechanisms involved are poorly understood. Importantly it appears that at least some of AIM2 functions during tumorigenesis may not rely on the assembly of typical inflammasome platforms.

\section{CONCLUSIONS AND PERSPECTIVES}

Inflammasomes are key players in the regulation of cellular stress and immune responses. The molecular mechanisms governing initiation and assembly of inflammasomes are still poorly understood. While most inflammasomes described so far respond to wellcharacterized stimuli, how the signal is transmitted to the scaffolding receptor is mostly unknown. The ligands of most inflammasome complexes are still unclear or poorly characterized except for AIM2 that is a bona fide DNA detector. DNA is ubiquitous; therefore, AIM2 can be considered a sensor of pathogens when it detects microbial DNA. However when it detects mislocalized self-DNA, it functions as a sensor of altered self. It 
behaves as a guardian of cellular integrity that can responds to perturbations and loss of cellular homeostasis such as, for example, disruption of nuclear envelope integrity. This raises several questions that remain to be elucidated. Recent studies have demonstrated that AIM2 detects self-DNA. However, how this detection is regulated to achieve specificity in order to detect pathogen and stress conditions and how this contributes to immune responses is still unclear. During mitosis, the phosphorylation of nuclear lamins causes the nuclear envelope to disintegrate into small membrane vesicles. The disruption of the nuclear compartment exposes chromosomal DNA to cytosolic components. Whether DNA packed within chromosomes can engage AIM2 is debated. However it is likely that during mitosis dsDNA is exposed and mechanisms are in place to avoid aberrant AIM2 activation. This has been proposed for the NLRP3 inflammasome. NLRP3 activation requires the binding of NEK7, a kinase involved in cell cycle. It was hypothesized that NEK7 function and regulation during cell cycle, limits its availability to engage NLRP3, thereby dampening NLRP3 activation (167). Thus, this mechanism limits NLRP3 activation during cell cycle and restrict it to cells in interphase. Why NLRP3 activation is blocked during cell cycle progression is currently unknown, and NEK7 does not affect AIM2 activation. However it is tempting to speculate that similar mechanisms, yet to be described, may function as a safeguard to limit aberrant AIM2 activation in cells undergoing cell division.

The function of AIM2 is also highly controlled by regulation of its expression and subcellular localization that may vary depending on several factors including the activation of other DNA sensors, the overall inflammatory status, and the tissues involved. Moreover the contribution of AIM2 regulators such as POP3, p202 and TRIM11 are still poorly 
understood and need to be evaluated in physiological models (155). It would be important to understand the contribution of these regulators to the modulation of the intensity and duration of the response as well as their possible role as safeguard mechanisms limiting aberrant detection of self-DNA.

AIM2 has been mostly studied in cells of the immune system or in the context of infection and immune deregulation, however its role in other tissues awaits dedidcated studies. Evidences suggests, for example that, in neurons, AIM2 may contribute neuronal morphology and influence anxiety and memory in mice (115).

Another important question that is emerging is the contribution of AIM2 to inflammasome independent processes. This has been shown mostly in cancer cells, but may also contribute to some of the phenotype observed in AIM2 deficient mice.

It is foreseeable that these questions will uncover new functions of AIM2 and provide a better understanding of the mechanisms by which it promotes immunity and contributes to diseases, in particular those with signs of autoinflammation and autoimmunity.

\section{ACKOWLEDGEMENTS}

FM is supported by supported by a grant from the Swiss National Science Foundation (310030_173152).

\section{CONFLICT OF INTEREST}

The author discloses no conflicts of interest.

\section{REFERENCES}

1. Rock KL, Latz E, Ontiveros F, Kono H. The sterile inflammatory response. Annual review of immunology.2010;28:321-342. 
2. Liston A, Masters SL. Homeostasis-altering molecular processes as mechanisms of inflammasome activation. Nature reviews Immunology.2017;17:208-214.

3. Gay NJ, Symmons MF, Gangloff M, Bryant CE. Assembly and localization of Tolllike receptor signalling complexes. Nature reviews Immunology.2014;14:546-558.

4. Albrecht M, Choubey D, Lengauer T. The HIN domain of IFI-200 proteins consists of two OB folds. Biochemical and biophysical research communications.2005;327:679687.

5. Ludlow LE, Johnstone RW, Clarke CJ. The HIN-200 family: more than interferoninducible genes? Experimental cell research.2005;308:1-17.

6. Shaw N, Liu ZJ. Role of the HIN domain in regulation of innate immune responses. Molecular and cellular biology.2014;34:2-15.

7. Martinon F, Hofmann K, Tschopp J. The pyrin domain: a possible member of the death domain-fold family implicated in apoptosis and inflammation. Current biology : CB.2001;11:R118-120.

8. Pawlowski K, Pio F, Chu Z, Reed JC, Godzik A. PAAD - a new protein domain associated with apoptosis, cancer and autoimmune diseases. Trends in biochemical sciences.2001;26:85-87.

9. Burckstummer T, et al. An orthogonal proteomic-genomic screen identifies AIM2 as a cytoplasmic DNA sensor for the inflammasome. Nature immunology.2009;10:266272.

10. Fernandes-Alnemri T, Yu JW, Datta P, Wu J, Alnemri ES. AIM2 activates the inflammasome and cell death in response to cytoplasmic DNA. Nature.2009;458:509513.

11. Hornung V, et al. AIM2 recognizes cytosolic dsDNA and forms a caspase-1-activating inflammasome with ASC. Nature.2009;458:514-518.

12. Cridland JA, et al. The mammalian PYHIN gene family: phylogeny, evolution and expression. BMC evolutionary biology.2012;12:140.

13. Brunette RL, Young JM, Whitley DG, Brodsky IE, Malik HS, Stetson DB. Extensive evolutionary and functional diversity among mammalian AIM2-like receptors. The Journal of experimental medicine.2012;209:1969-1983.

14. Deschamps M, et al. Genomic Signatures of Selective Pressures and Introgression from Archaic Hominins at Human Innate Immunity Genes. American journal of human genetics.2016;98:5-21.

15. Tschopp J, Martinon F, Burns K. NALPs: a novel protein family involved in inflammation. Nature reviews Molecular cell biology.2003;4:95-104.

16. Cagliani R, et al. Ancient and recent selective pressures shaped genetic diversity at AIM2-like nucleic acid sensors. Genome biology and evolution.2014;6:830-845.

17. Ahn M, Cui J, Irving AT, Wang LF. Unique Loss of the PYHIN Gene Family in Bats Amongst Mammals: Implications for Inflammasome Sensing. Scientific reports.2016;6:21722. 
18. DeYoung KL, et al. Cloning a novel member of the human interferon-inducible gene family associated with control of tumorigenicity in a model of human melanoma. Oncogene.1997;15:453-457.

19. Choubey D, Walter S, Geng Y, Xin H. Cytoplasmic localization of the interferoninducible protein that is encoded by the AIM2 (absent in melanoma) gene from the 200-gene family. FEBS letters.2000;474:38-42.

20. Samanta H, Pravtcheva DD, Ruddle FH, Lengyel P. Chromosomal location of mouse gene 202 which is induced by interferons and specifies a $56.5 \mathrm{kD}$ protein. Journal of interferon research.1984;4:295-300.

21. Kingsmore SF, Snoddy J, Choubey D, Lengyel P, Seldin MF. Physical mapping of a family of interferon-activated genes, serum amyloid P-component, and alpha-spectrin on mouse chromosome 1. Immunogenetics.1989;30:169-174.

22. Landolfo S, Gariglio M, Gribaudo G, Lembo D. The Ifi 200 genes: an emerging family of IFN-inducible genes. Biochimie.1998;80:721-728.

23. Lassig C, Hopfner KP. Discrimination of cytosolic self and non-self RNA by RIG-Ilike receptors. The Journal of biological chemistry.2017;292:9000-9009.

24. Ablasser A, Gulen MF. The role of cGAS in innate immunity and beyond. Journal of molecular medicine.2016;94:1085-1093.

25. Labzin LI, Lauterbach MA, Latz E. Interferons and inflammasomes: Cooperation and counterregulation in disease. The Journal of allergy and clinical immunology.2016;138:37-46.

26. Martinon F, Mayor A, Tschopp J. The inflammasomes: guardians of the body. Annual review of immunology.2009;27:229-265.

27. Man SM, et al. IRGB10 Liberates Bacterial Ligands for Sensing by the AIM2 and Caspase-11-NLRP3 Inflammasomes. Cell.2016;167:382-396 e317.

28. Martinon F, Burns K, Tschopp J. The inflammasome: a molecular platform triggering activation of inflammatory caspases and processing of proIL-beta. Molecular cell.2002;10:417-426.

29. Mace PD, Riedl SJ, Salvesen GS. Caspase enzymology and activation mechanisms. Methods in enzymology.2014;544:161-178.

30. Shakeri R, Kheirollahi A, Davoodi J. Apaf-1: Regulation and function in cell death. Biochimie.2017;135:111-125.

31. Acehan D, Jiang X, Morgan DG, Heuser JE, Wang X, Akey CW. Three-dimensional structure of the apoptosome: implications for assembly, procaspase-9 binding, and activation. Molecular cell.2002;9:423-432.

32. Agostini L, Martinon F, Burns K, McDermott MF, Hawkins PN, Tschopp J. NALP3 forms an IL-1beta-processing inflammasome with increased activity in Muckle-Wells autoinflammatory disorder. Immunity.2004;20:319-325.

33. He Y, Hara H, Nunez G. Mechanism and Regulation of NLRP3 Inflammasome Activation. Trends in biochemical sciences.2016;41:1012-1021. 
34. Cai $X$, et al. Prion-like polymerization underlies signal transduction in antiviral immune defense and inflammasome activation. Cell.2014;156:1207-1222.

35. Cai X, Xu H, Chen ZJ. Prion-Like Polymerization in Immunity and Inflammation. Cold Spring Harbor perspectives in biology.2017;9.

36. $\mathrm{Lu} \mathrm{A}$, et al. Unified polymerization mechanism for the assembly of ASC-dependent inflammasomes. Cell.2014;156:1193-1206.

37. Morrone SR, Matyszewski M, Yu X, Delannoy M, Egelman EH, Sohn J. Assemblydriven activation of the AIM2 foreign-dsDNA sensor provides a polymerization template for downstream ASC. Nature communications.2015;6:7827.

38. Dick MS, Sborgi L, Ruhl S, Hiller S, Broz P. ASC filament formation serves as a signal amplification mechanism for inflammasomes. Nature communications.2016;7:11929.

39. Dinarello CA. Immunological and inflammatory functions of the interleukin-1 family. Annual review of immunology.2009;27:519-550.

40. Dinarello CA. Infection, fever, and exogenous and endogenous pyrogens: some concepts have changed. Journal of endotoxin research.2004;10:201-222.

41. Martinon F, Tschopp J. NLRs join TLRs as innate sensors of pathogens. Trends in immunology.2005;26:447-454.

42. Cookson BT, Brennan MA. Pro-inflammatory programmed cell death. Trends in microbiology.2001;9:113-114.

43. Man SM, Karki R, Kanneganti TD. Molecular mechanisms and functions of pyroptosis, inflammatory caspases and inflammasomes in infectious diseases. Immunological reviews.2017;277:61-75.

44. Hersh D, Monack DM, Smith MR, Ghori N, Falkow S, Zychlinsky A. The Salmonella invasin SipB induces macrophage apoptosis by binding to caspase-1. Proceedings of the National Academy of Sciences of the United States of America.1999;96:23962401.

45. Hilbi H, et al. Shigella-induced apoptosis is dependent on caspase-1 which binds to IpaB. The Journal of biological chemistry.1998;273:32895-32900.

46. Liu T, et al. Single-cell imaging of caspase-1 dynamics reveals an all-or-none inflammasome signaling response. Cell reports.2014;8:974-982.

47. Sagulenko V, et al. AIM2 and NLRP3 inflammasomes activate both apoptotic and pyroptotic death pathways via ASC. Cell death and differentiation.2013;20:1149-1160.

48. Weinlich R, Oberst A, Beere HM, Green DR. Necroptosis in development, inflammation and disease. Nature reviews Molecular cell biology.2017;18:127-136.

49. Holler N, et al. Fas triggers an alternative, caspase-8-independent cell death pathway using the kinase RIP as effector molecule. Nature immunology.2000;1:489-495.

50. Aglietti RA, Dueber EC. Recent Insights into the Molecular Mechanisms Underlying Pyroptosis and Gasdermin Family Functions. Trends in immunology.2017. 
51. Abhishek A, Valdes AM, Doherty M. Low omega-3 fatty acid levels associate with frequent gout attacks: a case control study. Annals of the rheumatic diseases.2016;75:784-785.

52. Kayagaki N, et al. Caspase-11 cleaves gasdermin D for non-canonical inflammasome signalling. Nature.2015;526:666-671.

53. Muruve DA, et al. The inflammasome recognizes cytosolic microbial and host DNA and triggers an innate immune response. Nature.2008;452:103-107.

54. Roberts TL, et al. HIN-200 proteins regulate caspase activation in response to foreign cytoplasmic DNA. Science.2009;323:1057-1060.

55. Hemmi H, et al. A Toll-like receptor recognizes bacterial DNA. Nature.2000;408:740745 .

56. Kim T, et al. Aspartate-glutamate-alanine-histidine box motif (DEAH)/RNA helicase A helicases sense microbial DNA in human plasmacytoid dendritic cells. Proc Natl Acad Sci U S A.2010;107:15181-15186.

57. Zhang $\mathrm{X}$, et al. Cutting edge: $\mathrm{Ku} 70$ is a novel cytosolic DNA sensor that induces type III rather than type I IFN. J Immunol.2011;186:4541-4545.

58. Dempsey A, Bowie AG. Innate immune recognition of DNA: A recent history. Virology.2015;479-480:146-152.

59. Ishikawa H, Ma Z, Barber GN. STING regulates intracellular DNA-mediated, type I interferon-dependent innate immunity. Nature.2009;461:788-792.

60. Gao D, et al. Cyclic GMP-AMP synthase is an innate immune sensor of HIV and other retroviruses. Science.2013;341:903-906.

61. Wu J, et al. Cyclic GMP-AMP is an endogenous second messenger in innate immune signaling by cytosolic DNA. Science.2013;339:826-830.

62. Zhang Z, Yuan B, Bao M, Lu N, Kim T, Liu YJ. The helicase DDX41 senses intracellular DNA mediated by the adaptor STING in dendritic cells. Nat Immunol.2011;12:959-965.

63. Ferguson BJ, Mansur DS, Peters NE, Ren H, Smith GL. DNA-PK is a DNA sensor for IRF-3-dependent innate immunity. Elife.2012;1:e00047.

64. Kondo T, et al. DNA damage sensor MRE11 recognizes cytosolic double-stranded DNA and induces type I interferon by regulating STING trafficking. Proc Natl Acad Sci U S A.2013;110:2969-2974.

65. Takaoka A, et al. DAI (DLM-1/ZBP1) is a cytosolic DNA sensor and an activator of innate immune response. Nature.2007;448:501-505.

66. Wang Z, et al. Regulation of innate immune responses by DAI (DLM-1/ZBP1) and other DNA-sensing molecules. Proc Natl Acad Sci U S A.2008;105:5477-5482.

67. Unterholzner L, et al. IFI16 is an innate immune sensor for intracellular DNA. Nat Immunol.2010;11:997-1004. 
68. Jin T, et al. Structures of the HIN domain:DNA complexes reveal ligand binding and activation mechanisms of the AIM2 inflammasome and IFI16 receptor.

Immunity.2012;36:561-571.

69. Sung MW, Watts T, Li P. Crystallographic characterization of mouse AIM2 HIN-200 domain bound to a $15 \mathrm{bp}$ and an $18 \mathrm{bp}$ double-stranded DNA. Acta crystallographica Section F, Structural biology and crystallization communications.2012;68:1081-1084.

70. Li H, Wang J, Wang J, Cao LS, Wang ZX, Wu JW. Structural mechanism of DNA recognition by the p202 HINa domain: insights into the inhibition of Aim2-mediated inflammatory signalling. Acta crystallographica Section F, Structural biology communications.2014;70:21-29.

71. Man SM, Karki R, Kanneganti TD. AIM2 inflammasome in infection, cancer, and autoimmunity: Role in DNA sensing, inflammation, and innate immunity. Eur J Immunol.2016;46:269-280.

72. Fernandes-Alnemri T, et al. The AIM2 inflammasome is critical for innate immunity to Francisella tularensis. Nature immunology.2010;11:385-393.

73. Rathinam VA, et al. The AIM2 inflammasome is essential for host defense against cytosolic bacteria and DNA viruses. Nature immunology.2010;11:395-402.

74. Jones JW, et al. Absent in melanoma 2 is required for innate immune recognition of Francisella tularensis. Proceedings of the National Academy of Sciences of the United States of America.2010;107:9771-9776.

75. Warren SE, et al. Cutting edge: Cytosolic bacterial DNA activates the inflammasome via Aim2. Journal of immunology.2010;185:818-821.

76. Meunier E, et al. Guanylate-binding proteins promote activation of the AIM2 inflammasome during infection with Francisella novicida. Nature immunology.2015;16:476-484.

77. Man SM, et al. The transcription factor IRF1 and guanylate-binding proteins target activation of the AIM2 inflammasome by Francisella infection. Nature immunology.2015;16:467-475.

78. Reinholz M, et al. HPV16 activates the AIM2 inflammasome in keratinocytes. Arch Dermatol Res.2013;305:723-732.

79. Torii Y, Kawada JI, Murata T, Yoshiyama H, Kimura H, Ito Y. Epstein-Barr virus infection-induced inflammasome activation in human monocytes. PLoS One.2017;12:e0175053.

80. Schattgen SA, Gao G, Kurt-Jones EA, Fitzgerald KA. Cutting Edge: DNA in the Lung Microenvironment during Influenza Virus Infection Tempers Inflammation by Engaging the DNA Sensor AIM2. J Immunol.2016;196:29-33.

81. Zhang H, et al. AIM2 Inflammasome Is Critical for Influenza-Induced Lung Injury and Mortality. Journal of immunology.2017;198:4383-4393.

82. Hetzer MW. The nuclear envelope. Cold Spring Harbor perspectives in biology.2010;2:a000539. 
83. Dobrzynska A, Gonzalo S, Shanahan C, Askjaer P. The nuclear lamina in health and disease. Nucleus.2016;7:233-248.

84. Davidson PM, Lammerding J. Broken nuclei--lamins, nuclear mechanics, and disease. Trends in cell biology.2014;24:247-256.

85. de Noronha CM, et al. Dynamic disruptions in nuclear envelope architecture and integrity induced by HIV-1 Vpr. Science.2001;294:1105-1108.

86. De Vos WH, et al. Repetitive disruptions of the nuclear envelope invoke temporary loss of cellular compartmentalization in laminopathies. Human molecular genetics.2011;20:4175-4186.

87. Prokocimer M, Margalit A, Gruenbaum Y. The nuclear lamina and its proposed roles in tumorigenesis: projection on the hematologic malignancies and future targeted therapy. Journal of structural biology.2006;155:351-360.

88. Zink D, Fischer AH, Nickerson JA. Nuclear structure in cancer cells. Nature reviews Cancer.2004;4:677-687.

89. Raab M, et al. ESCRT III repairs nuclear envelope ruptures during cell migration to limit DNA damage and cell death. Science.2016;352:359-362.

90. Hatch E, Hetzer M. Breaching the nuclear envelope in development and disease. The Journal of cell biology.2014;205:133-141.

91. Webster BM, Lusk CP. Border Safety: Quality Control at the Nuclear Envelope. Trends in cell biology.2016;26:29-39.

92. Denais $\mathrm{CM}$, et al. Nuclear envelope rupture and repair during cancer cell migration. Science.2016;352:353-358.

93. Di Micco A, et al. AIM2 inflammasome is activated by pharmacological disruption of nuclear envelope integrity. Proceedings of the National Academy of Sciences of the United States of America.2016;113:E4671-4680.

94. $\mathrm{Hu} \mathrm{B}$, et al. The DNA-sensing AIM2 inflammasome controls radiation-induced cell death and tissue injury. Science.2016;354:765-768.

95. Qwarnstrom EE, Page RC, Gillis S, Dower SK. Binding, internalization, and intracellular localization of interleukin-1 beta in human diploid fibroblasts. The Journal of biological chemistry.1988;263:8261-8269.

96. Sborgi L, et al. GSDMD membrane pore formation constitutes the mechanism of pyroptotic cell death. The EMBO journal.2016;35:1766-1778.

97. Lian Q, et al. Chemotherapy-induced intestinal inflammatory responses are mediated by exosome secretion of double-strand DNA via AIM2 inflammasome activation. Cell research.2017;27:784-800.

98. Suschak JJ, Wang S, Fitzgerald KA, Lu S. Identification of Aim2 as a sensor for DNA vaccines. Journal of immunology.2015;194:630-636.

99. Ishii KJ, et al. TANK-binding kinase-1 delineates innate and adaptive immune responses to DNA vaccines. Nature.2008;451:725-729. 
100.Corrales L, Woo SR, Williams JB, McWhirter SM, Dubensky TW, Jr., Gajewski TF. Antagonism of the STING Pathway via Activation of the AIM2 Inflammasome by Intracellular DNA. J Immunol.2016;196:3191-3198.

101.Wang Y, et al. Inflammasome Activation Triggers Caspase-1-Mediated Cleavage of cGAS to Regulate Responses to DNA Virus Infection. Immunity.2017;46:393-404.

102.Chai D, Yue Y, Xu W, Dong C, Xiong S. Mucosal co-immunization with AIM2 enhances protective SIgA response and increases prophylactic efficacy of chitosanDNA vaccine against coxsackievirus B3-induced myocarditis. Hum Vaccin Immunother.2014;10:1284-1294.

103.Chai D, Yue Y, Xu W, Dong C, Xiong S. AIM2 co-immunization favors specific multifunctional CD8(+) T cell induction and ameliorates coxsackievirus B3-induced chronic myocarditis. Antiviral Res.2015;119:68-77.

104.Yin L, Chai D, Yue Y, Dong C, Xiong S. AIM2 Co-immunization with VP1 Is Associated with Increased Memory CD8 T Cells and Mounts Long Lasting Protection against Coxsackievirus B3 Challenge. Front Cell Infect Microbiol.2017;7:247.

105.Masters SL, Simon A, Aksentijevich I, Kastner DL. Horror autoinflammaticus: the molecular pathophysiology of autoinflammatory disease $(*)$. Annual review of immunology.2009;27:621-668.

106.Stetson DB, Ko JS, Heidmann T, Medzhitov R. Trex1 prevents cell-intrinsic initiation of autoimmunity. Cell.2008;134:587-598.

107.Kawane K, Motani K, Nagata S. DNA degradation and its defects. Cold Spring Harbor perspectives in biology.2014;6.

108.Kaminski JJ, Schattgen SA, Tzeng TC, Bode C, Klinman DM, Fitzgerald KA. Synthetic oligodeoxynucleotides containing suppressive TTAGGG motifs inhibit AIM2 inflammasome activation. Journal of immunology.2013;191:3876-3883.

109.Dorfleutner A, Chu L, Stehlik C. Inhibiting the inflammasome: one domain at a time. Immunological reviews.2015;265:205-216.

110.Johnston JB, et al. A poxvirus-encoded pyrin domain protein interacts with ASC-1 to inhibit host inflammatory and apoptotic responses to infection. Immunity.2005;23:587-598.

111. Khare S, et al. The PYRIN domain-only protein POP3 inhibits ALR inflammasomes and regulates responses to infection with DNA viruses. Nature immunology.2014;15:343-353.

112.Martinon F, Tschopp J. Inflammatory caspases and inflammasomes: master switches of inflammation. Cell death and differentiation.2007;14:10-22.

113.Yin Q, et al. Molecular mechanism for $\mathrm{p} 202$-mediated specific inhibition of AIM2 inflammasome activation. Cell reports.2013;4:327-339.

114. Ru H, et al. Structural basis for termination of AIM2-mediated signaling by p202. Cell research.2013;23:855-858. 
115.Wu PJ, Liu HY, Huang TN, Hsueh YP. AIM 2 inflammasomes regulate neuronal morphology and influence anxiety and memory in mice. Scientific reports.2016;6:32405.

116.Yang J, Liu Z, Xiao TS. Post-translational regulation of inflammasomes. Cellular \& molecular immunology.2017;14:65-79.

117.Shah S, et al. Cutting edge: Mycobacterium tuberculosis but not nonvirulent mycobacteria inhibits IFN-beta and AIM2 inflammasome-dependent IL-1beta production via its ESX-1 secretion system. Journal of immunology.2013;191:35143518.

118.Wassermann R, et al. Mycobacterium tuberculosis Differentially Activates cGAS- and Inflammasome-Dependent Intracellular Immune Responses through ESX-1. Cell host \& microbe.2015;17:799-810.

119.Crane DD, Bauler TJ, Wehrly TD, Bosio CM. Mitochondrial ROS potentiates indirect activation of the AIM2 inflammasome. Front Microbiol.2014;5:438.

120.Huang MT, et al. Deletion of ripA alleviates suppression of the inflammasome and MAPK by Francisella tularensis. J Immunol.2010;185:5476-5485.

121.Ulland TK, et al. Cutting edge: mutation of Francisella tularensis mviN leads to increased macrophage absent in melanoma 2 inflammasome activation and a loss of virulence. J Immunol.2010;185:2670-2674.

122.Ge J, Gong YN, Xu Y, Shao F. Preventing bacterial DNA release and absent in melanoma 2 inflammasome activation by a Legionella effector functioning in membrane trafficking. Proceedings of the National Academy of Sciences of the United States of America.2012;109:6193-6198.

123.Sampson TR, et al. A CRISPR-Cas system enhances envelope integrity mediating antibiotic resistance and inflammasome evasion. Proceedings of the National Academy of Sciences of the United States of America.2014;111:11163-11168.

124.Brodmann M, Dreier RF, Broz P, Basler M. Francisella requires dynamic type VI secretion system and $\mathrm{ClpB}$ to deliver effectors for phagosomal escape. Nat Commun.2017;8:15853.

125.Eichholz K, et al. Immune-Complexed Adenovirus Induce AIM2-Mediated Pyroptosis in Human Dendritic Cells. PLoS pathogens.2016;12:e1005871.

126.Zhen J, et al. AIM2 mediates inflammation-associated renal damage in hepatitis B virus-associated glomerulonephritis by regulating caspase-1, IL-1beta, and IL-18. Mediators Inflamm.2014;2014:190860.

127.Huang Y, et al. Interaction between HCMV pUL83 and human AIM2 disrupts the activation of the AIM2 inflammasome. Virology journal.2017;14:34.

128.Ekchariyawat $\mathrm{P}$, et al. Inflammasome signaling pathways exert antiviral effect against Chikungunya virus in human dermal fibroblasts. Infect Genet Evol.2015;32:401-408. 
129. Yogarajah T, Ong KC, Perera D, Wong KT. AIM2 Inflammasome-Mediated Pyroptosis in Enterovirus A71-Infected Neuronal Cells Restricts Viral Replication. Sci Rep.2017;7:5845.

130.Karki R, et al. Concerted activation of the AIM2 and NLRP3 inflammasomes orchestrates host protection against Aspergillus infection. Cell host \& microbe.2015;17:357-368.

131.Kalantari P, et al. Dual engagement of the NLRP3 and AIM2 inflammasomes by plasmodium-derived hemozoin and DNA during malaria. Cell reports.2014;6:196-210.

132. Moreira RB, et al. AIM2 inflammasome is associated with disease severity in tegumentary leishmaniasis caused by Leishmania (V.) braziliensis. Parasite immunology.2017;39.

133.Ratsimandresy RA, Indramohan M, Dorfleutner A, Stehlik C. The AIM2 inflammasome is a central regulator of intestinal homeostasis through the IL-18/IL22/STAT3 pathway. Cellular \& molecular immunology.2017;14:127-142.

134.Man SM, Karki R, Kanneganti TD. DNA-sensing inflammasomes: regulation of bacterial host defense and the gut microbiota. Pathogens and disease.2016;74:ftw028.

135. Hu GQ, et al. AIM2 contributes to the maintenance of intestinal integrity via Akt and protects against Salmonella mucosal infection. Mucosal immunology.2016;9:13301339.

136.Hu S, et al. The DNA Sensor AIM2 Maintains Intestinal Homeostasis via Regulation of Epithelial Antimicrobial Host Defense. Cell reports.2015;13:1922-1936.

137. Vanhove W, et al. Strong Upregulation of AIM2 and IFI16 Inflammasomes in the Mucosa of Patients with Active Inflammatory Bowel Disease. Inflammatory bowel diseases.2015;21:2673-2682.

138.McDermott MF, et al. Germline mutations in the extracellular domains of the $55 \mathrm{kDa}$ TNF receptor, TNFR1, define a family of dominantly inherited autoinflammatory syndromes. Cell.1999;97:133-144.

139.Stoffels M, Kastner DL. Old Dogs, New Tricks: Monogenic Autoinflammatory Disease Unleashed. Annual review of genomics and human genetics.2016;17:245-272.

140.Jesus AA, Goldbach-Mansky R. IL-1 blockade in autoinflammatory syndromes. Annual review of medicine.2014;65:223-244.

141.Martinon F, Petrilli V, Mayor A, Tardivel A, Tschopp J. Gout-associated uric acid crystals activate the NALP3 inflammasome. Nature.2006;440:237-241.

142.Jakobs C, Perner S, Hornung V. AIM2 Drives Joint Inflammation in a Self-DNA Triggered Model of Chronic Polyarthritis. PloS one.2015;10:e131702.

143. Baum R, et al. Cutting edge: AIM2 and endosomal TLRs differentially regulate arthritis and autoantibody production in DNase II-deficient mice. Journal of immunology.2015;194:873-877.

144.Dombrowski Y, et al. Cytosolic DNA triggers inflammasome activation in keratinocytes in psoriatic lesions. Science translational medicine.2011;3:82ra38. 
145. Choubey D, Panchanathan R. Absent in Melanoma 2 proteins in SLE. Clinical immunology.2017;176:42-48.

146.Zhang W, Cai Y, Xu W, Yin Z, Gao X, Xiong S. AIM2 facilitates the apoptotic DNAinduced systemic lupus erythematosus via arbitrating macrophage functional maturation. Journal of clinical immunology.2013;33:925-937.

147.Kim H, Sanchez GA, Goldbach-Mansky R. Insights from Mendelian Interferonopathies: Comparison of CANDLE, SAVI with AGS, Monogenic Lupus. Journal of molecular medicine.2016;94:1111-1127.

148. Rozzo SJ, et al. Evidence for an interferon-inducible gene, Ifi202, in the susceptibility to systemic lupus. Immunity.2001;15:435-443.

149.Gray EE, Winship D, Snyder JM, Child SJ, Geballe AP, Stetson DB. The AIM2-like Receptors Are Dispensable for the Interferon Response to Intracellular DNA. Immunity.2016;45:255-266.

150.Choubey D, Panchanathan R. Comment on "Deficient NLRP3 and AIM2 Inflammasome Function in Autoimmune NZB Mice". Journal of immunology.2015;195:4551-4552.

151.Sester DP, Stacey KJ. Response to Comment on "Deficient NLRP3 and AIM2 Inflammasome Function in Autoimmune NZB Mice". Journal of immunology.2015;195:4552-4553.

152.Sester DP, Stacey KJ. Correction: Response to Comment on "Deficient NLRP3 and AIM2 Inflammasome Function in Autoimmune NZB Mice". Journal of immunology.2016;197:676.

153.Elinav E, Nowarski R, Thaiss CA, Hu B, Jin C, Flavell RA. Inflammation-induced cancer: crosstalk between tumours, immune cells and microorganisms. Nat Rev Cancer.2013;13:759-771.

154.Karki R, Man SM, Kanneganti TD. Inflammasomes and Cancer. Cancer immunology research.2017;5:94-99.

155. Choubey D. Absent in melanoma 2 proteins in the development of cancer. Cellular and molecular life sciences : CMLS.2016;73:4383-4395.

156.Schulmann K, et al. HNPCC-associated small bowel cancer: clinical and molecular characteristics. Gastroenterology.2005;128:590-599.

157. Woerner SM, et al. The putative tumor suppressor AIM2 is frequently affected by different genetic alterations in microsatellite unstable colon cancers. Genes Chromosomes Cancer.2007;46:1080-1089.

158.Dihlmann S, et al. Lack of Absent in Melanoma 2 (AIM2) expression in tumor cells is closely associated with poor survival in colorectal cancer patients. Int $\mathbf{J}$

Cancer.2014;135:2387-2396.

159.Ponomareva L, et al. AIM2, an IFN-inducible cytosolic DNA sensor, in the development of benign prostate hyperplasia and prostate cancer. Mol Cancer Res.2013;11:1193-1202. 
160.Ma X, et al. Loss of AIM2 expression promotes hepatocarcinoma progression through activation of mTOR-S6K1 pathway. Oncotarget.2016;7:36185-36197.

161.Chen SL, et al. HBx-mediated decrease of AIM2 contributes to hepatocellular carcinoma metastasis. Mol Oncol.2017.

162.Man SM, et al. Critical Role for the DNA Sensor AIM2 in Stem Cell Proliferation and Cancer. Cell.2015;162:45-58.

163. Wilson JE, et al. Inflammasome-independent role of AIM2 in suppressing colon tumorigenesis via DNA-PK and Akt. Nature medicine.2015;21:906-913.

164.Farshchian M, et al. Tumor cell-specific AIM2 regulates growth and invasion of cutaneous squamous cell carcinoma. Oncotarget.2017.

165.Chen LC, et al. Tumour inflammasome-derived IL-1beta recruits neutrophils and improves local recurrence-free survival in EBV-induced nasopharyngeal carcinoma. EMBO Mol Med.2012;4:1276-1293.

166. Milutin Gasperov N, Farkas SA, Nilsson TK, Grce M. Epigenetic activation of immune genes in cervical cancer. Immunol Lett.2014;162:256-257.

167. Shi $\mathrm{H}$, et al. NLRP3 activation and mitosis are mutually exclusive events coordinated by NEK7, a new inflammasome component. Nature immunology.2016;17:250-258.

Figure 1: Activation of the AIM2 inflammasome. AIM2 is composed an N-terminal pyrin domain (PYD) and a C-terminal HIN domain. Both domains interact to keep the molecule auto-inhibited. Upon binding of dsDNA auto-inhibition is released leading to AIM2 oligomerization and the recruitment of the adaptor ASC via PYD-PYD interactions. ASC recruitment leads to polymerisation of the ASC protein leading to the formation of fibrilar super-structures. Caspase-1 is recruited to the complex via CARD-CARD interactions. Its oligomerization on the complex promotes its activity leading to the proteolytic maturation of caspase- 1 substrates such as the IL-1 $\beta$ precursor (pro-IL-1 $\beta$ ) that is processed into the biologically active IL-1 $\beta$.

\section{Figure 2: Mechanisms of Pyroptosis}


Assembly of inflammasomes, such as the AIM2 inflammasome triggers the activation of caspase-1 that can cleave GSDMD. This results in release of the GSDMD N-terminal (Nter) fragment. GSDM-Nter forms large pores in the plasma membrane that result in rapid loss of membrane integrity, the release of cellular content, including dangers signals such as uric acid, facilitates the release of other caspase-1 substrates such as IL$1 \beta$. eventually

pyroptotic

Figure 3: AIM2 Inflammasome activation upon destabilization of the nuclear envelope

Perturbations in the maturation of the Lamin A triggers transient alterations of NE structures and causes the transient exposure of self-DNA. Released DNA is detected by AIM2 leading to the assembly and activation of the AIM2 inflammasome.

\section{Figure 4: Regulation of AIM2 by decoy proteins.}

Decoy ALRs do not harbor the PYD and may interfere with AIM2 binding to DNA. These regulators may also impair AIM2 oligomerization. POPs are decoy ASC proteins that have only one PYD domain and are believed do inhibit PYD-PYD interactions between ASC and AIM2. They may function by interacting with AIM2, however some POPs may bind ASC and block its recruitment to the inflammasome scaffold. Decoy Caspase-1 (COPs) do 
not have the caspase domain and are believed to inhibit caspase- 1 recruitment to the Inflammasome by blocking CARD-CARD interactions. 

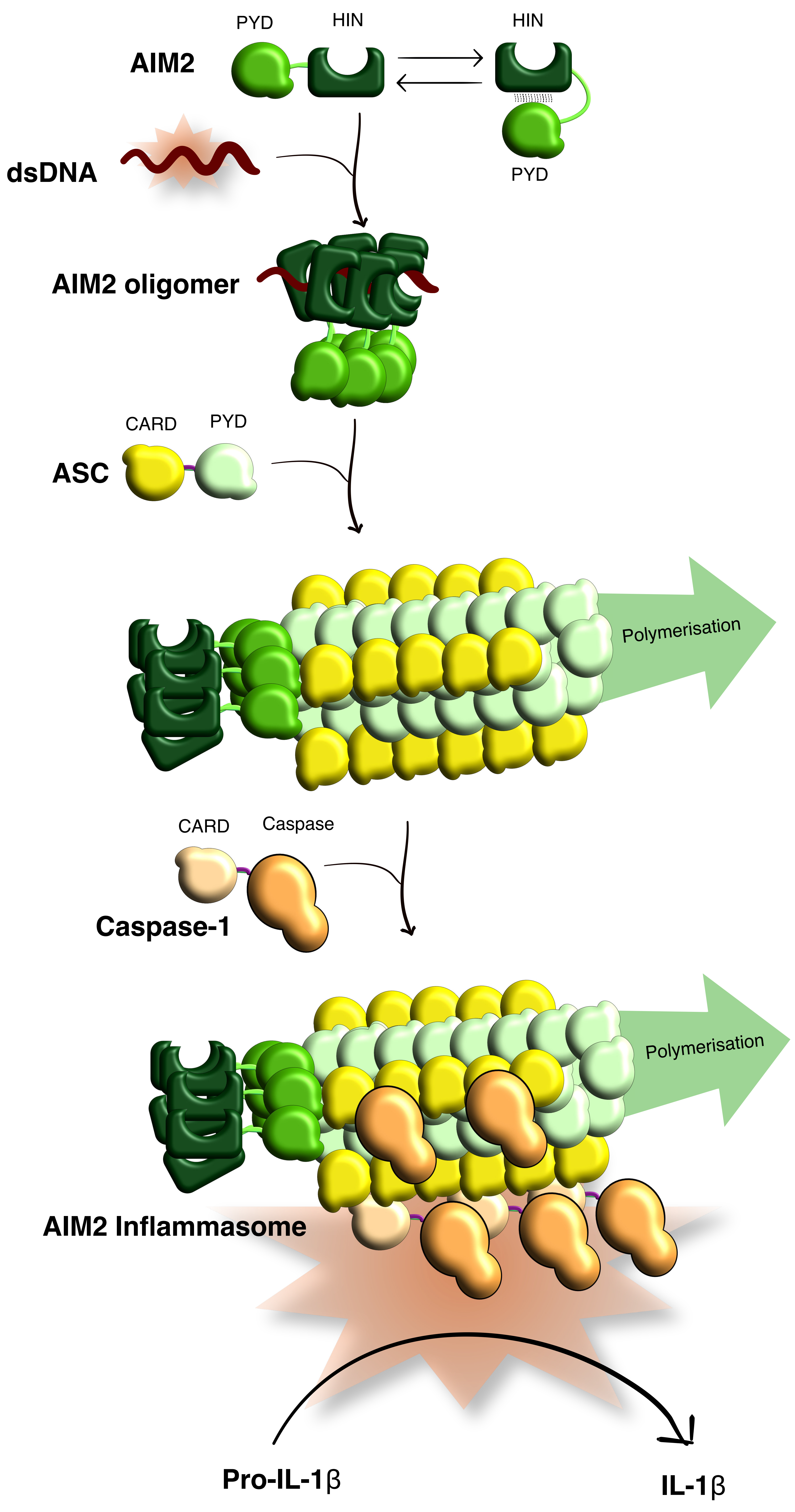


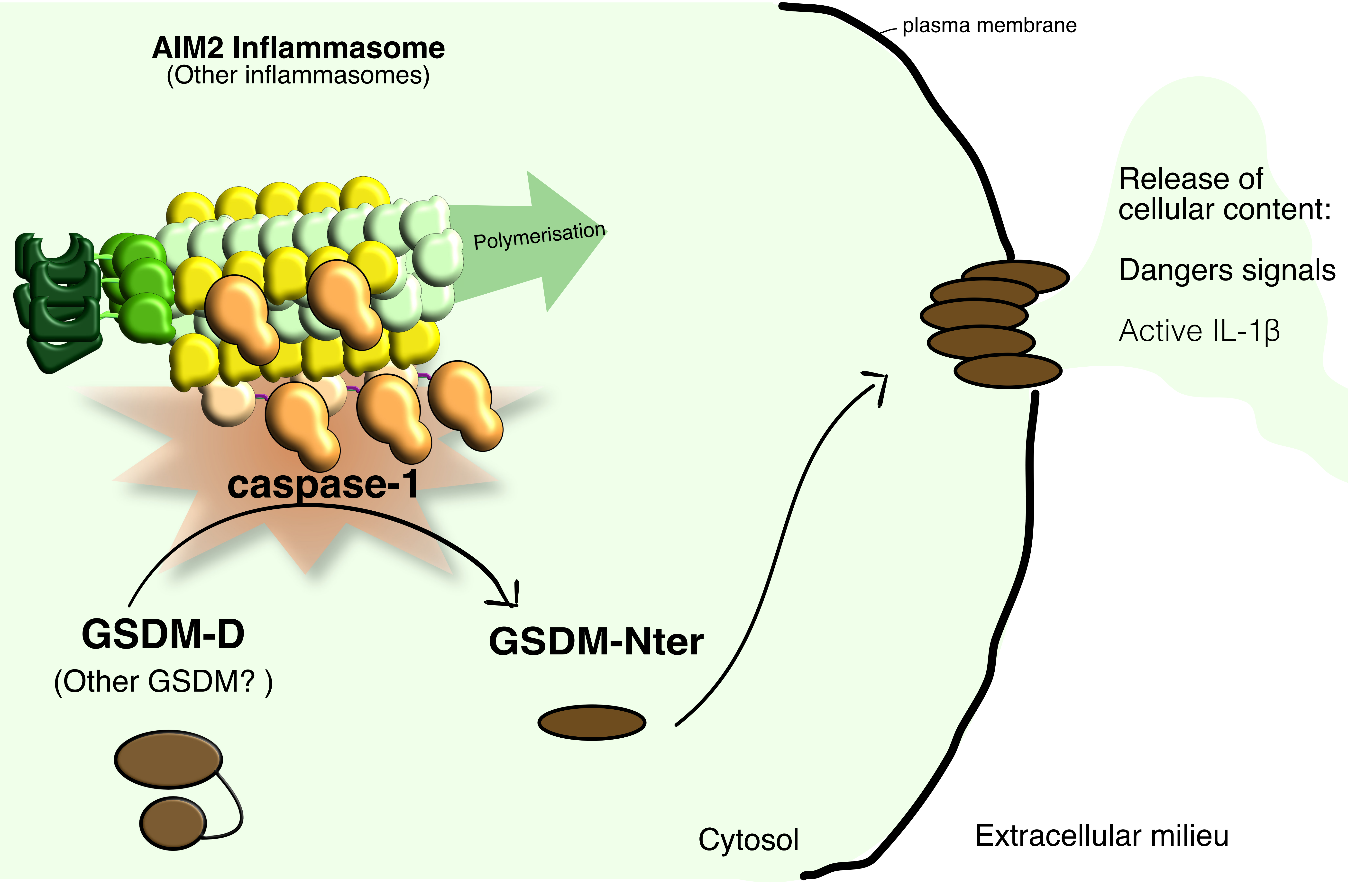


Perturbation of the nuclear lamina, Mechanical stress,

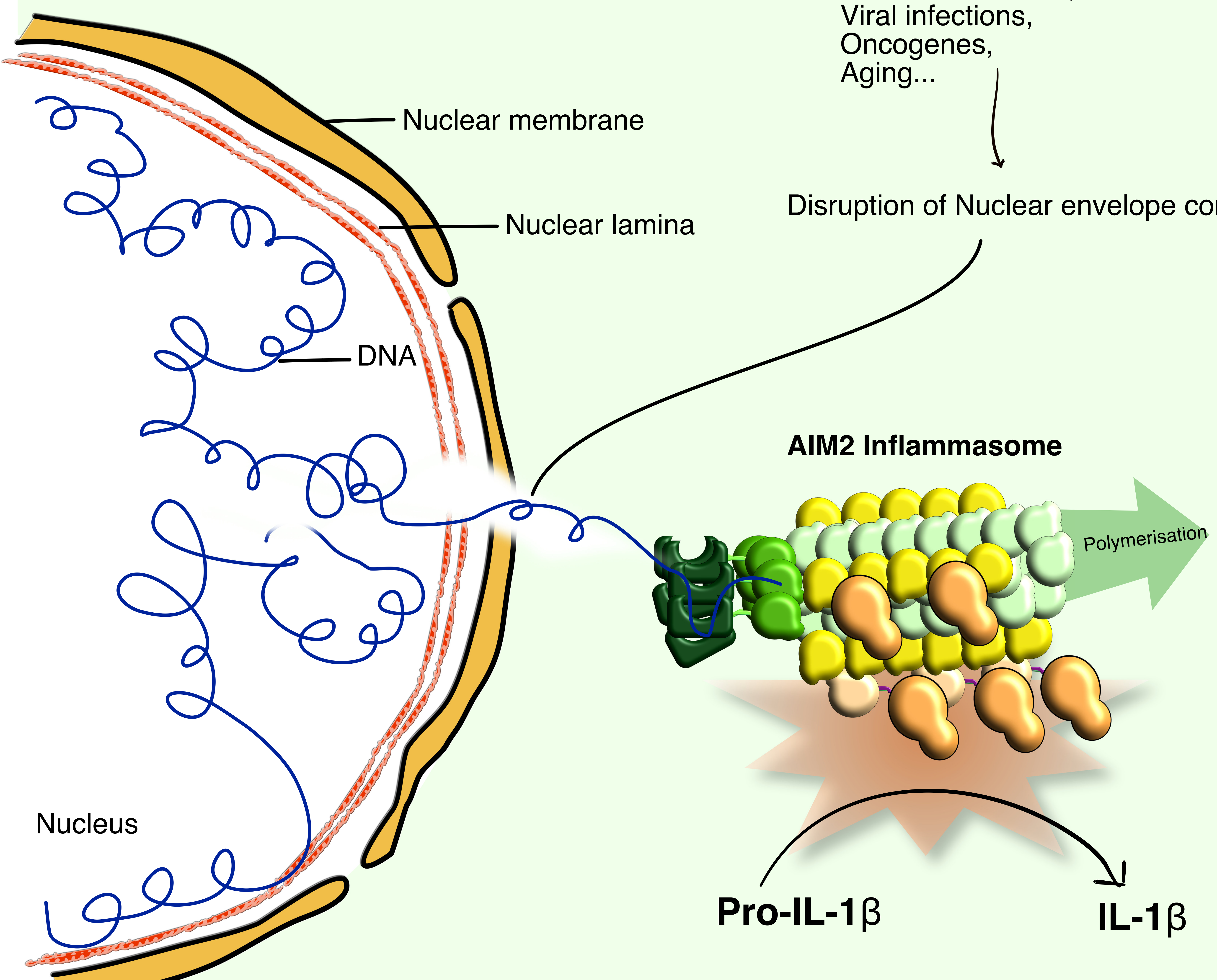

Cytosol 


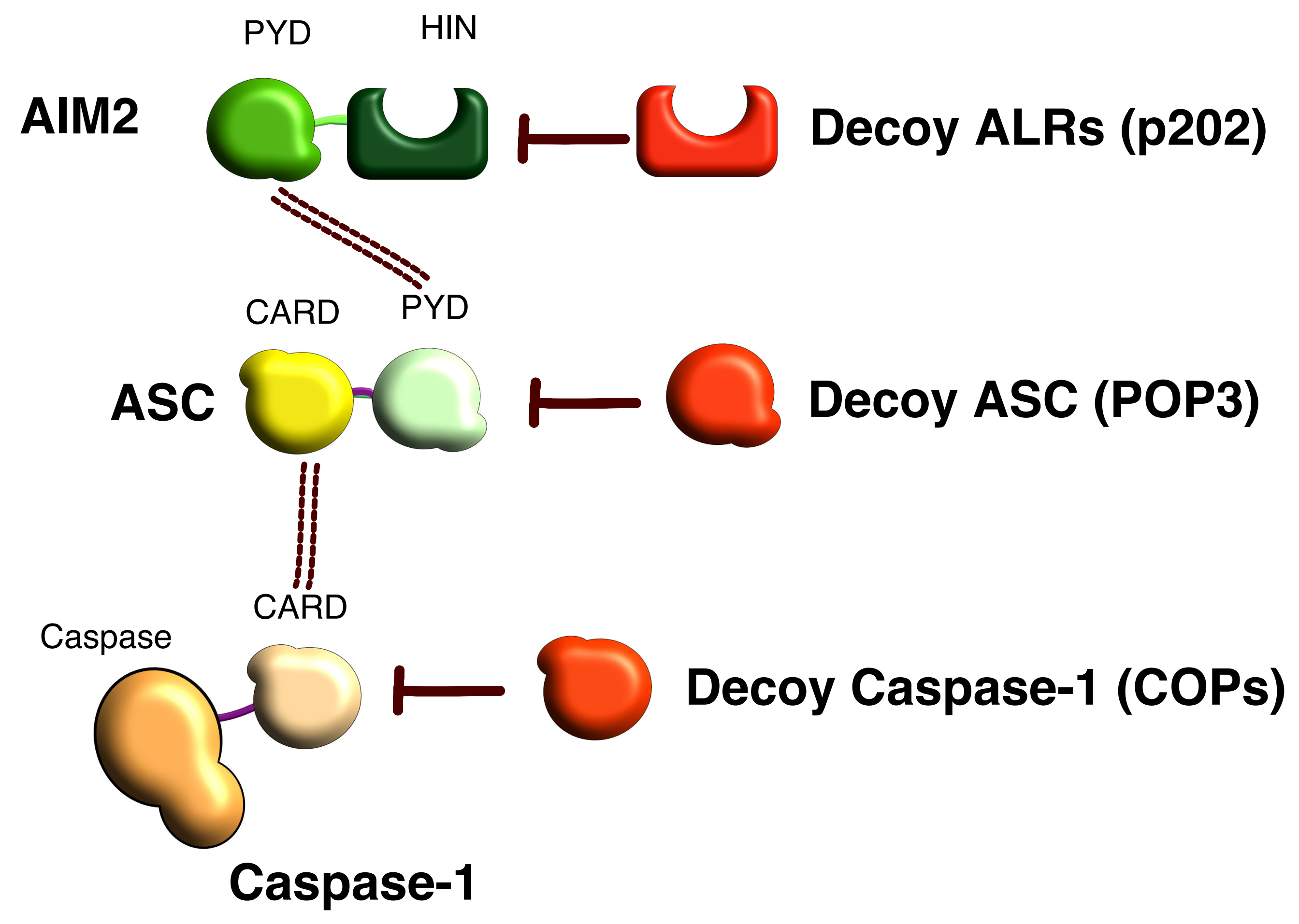

